\title{
Observations of Drizzle in Nocturnal Marine Stratocumulus
}

\author{
M. C. VANZANTEN \\ Department of Atmospheric Sciences, University of California, Los Angeles, Los Angeles, California, and Institute of Marine and \\ Atmospheric Research Utrecht, Utrecht University, Utrecht, Netherlands

\section{B. STEVENS} \\ Department of Atmospheric Sciences, University of California, Los Angeles, Los Angeles, California
}

\author{
G. VALI
}

Department of Atmospheric Science, University of Wyoming, Laramie, Wyoming

\author{
D. H. LENSCHOW
}

National Center for Atmospheric Research, ${ }^{*}$ Boulder, Colorado

(Manuscript received 17 October 2003, in final form 30 June 2004)

\begin{abstract}
In situ and radar data from the second field study of the Dynamics and Chemistry of Marine Stratocumulus (DYCOMS-II) have been used to study drizzle in stratocumulus. Measurements indicate that drizzle is prevalent. During five of seven analyzed flights precipitation was evident at the surface, and on roughly a third of the flights mean surface rates approached or exceeded $0.5 \mathrm{~mm} \mathrm{day}^{-1}$. Additional analysis of the structure and variability of drizzle indicates that the macroscopic (flight averaged) mean drizzle rates at cloud base scale with $H^{3} / N$ where $H$ is the flight-averaged cloud depth and $N$ the flight-averaged cloud droplet number concentration. To a lesser extent flight-to-flight variability in the mean drizzle rate also scales well with differences in the 11- and $4-\mu \mathrm{m}$ brightness temperatures, and the cloud-top effective radius. The structure of stratocumulus boundary layers with precipitation reaching the surface is also investigated, and a general picture emerges of large flight-averaged drizzle rates being manifested primarily through the emergence of intense pockets of precipitation. The characteristics of the drizzle spectrum in precipitating versus nonprecipitating regions of a particular cloud layer were mostly distinguished by the number of drizzle drops present, rather than a change in size of the median drizzle drop, or the breadth of the drizzle spectrum.
\end{abstract}

\section{Introduction}

Among the pantheon of processes involving stratocumulus, drizzle occupies a peculiar place. Despite observational evidence that it is commonplace, it is conspicuously absent in most of our conceptual and theoretical descriptions of stratocumulus.

Already during the late 1970 s and early 1980s measurements in stratocumulus (Brost et al. 1982; Nicholls 1984; Nicholls and Leighton 1986) showed that at times

\footnotetext{
* The National Center for Atmospheric Research is sponsored by the National Science Foundation.
}

Corresponding author address: M. C. vanZanten, Institute of Marine and Atmospheric Research Utrecht, Utrecht University, Princetonplein 5, 3584 CC Utrecht, Netherlands.

E-mail: M.C.vanZanten@phys.uu.nl the drizzle flux contributes significantly to the total water budget. Nicholls (1984) for instance showed that the gravitational settling of drizzle drops was commensurate with the turbulent flux, through the entire boundary layer, not just inside the cloud. Similar results were derived from an analysis of data collected during Atlantic Stratocumulus Transition Experiment (ASTEX) (e.g., Duynkerke et al. 1995; Frisch et al. 1995; Bretherton et al. 1995) and First International Satellite Cloud Climatology Project (ISCCP) Regional Experiment (FIRE; Austin et al. 1995) and can be inferred from measurements during the Southern Ocean Cloud Experiment (SOCEX; Boers et al. 1996, 1998) and data from an experiment off the coast of Oregon (Vali et al. 1998). In this paper, we present evidence from data collected during the Dynamics and Chemistry of Marine Stratocumulus II field study (DYCOMS-II) that drizzle may be even more prevalent than previously 
thought. In only two of the seven flights was there no evidence of drizzle at the sea surface and in two of the flights drizzle rates were substantial, making drizzle something more of a rule than an exception.

Despite the observational record, in the modeling community drizzle is often neglected or treated marginally [see e.g., the stratocumulus cases simulated by the Global Energy and Water Cycle Experiment (GEWEX) Cloud System Study (GCSS) working group 1; Duynkerke et al. 1999]. Of existing drizzle modeling work, most is concerned with either the formation of drizzle (e.g., Nicholls 1987; Feingold et al. 1996; Austin et al. 1995) or the influence of turbulence on the microphysics of stratocumulus clouds (Kogan et al. 1995). Both types of studies have a distinct microphysical perspective. Cloud macroscopic features and their relation to drizzle are less often studied. The feedback of drizzle on cloud dynamics has been dealt with in some onedimensional modeling studies (e.g., Albrecht 1989; Ackerman et al. 1993; Pincus and Baker 1994; Chen and Cotton 1987; Wang and Wang 1994), in which of course most of the important processes have to be parameterized. Stevens et al. (1998) is one of a few studies so far to utilize large eddy simulation to study how drizzle interacts with the turbulent structure of the PBL. By and large all of these studies provide support for the idea that drizzle can regulate cloudiness in important ways.

But in our theoretical development of the subject, the role of drizzle as trait d'union between cloud microphysics and cloud dynamics is still somewhat overlooked. Is this warranted? Should we think of drizzle as being an important process but yet of secondary significance so that neglecting it is justified? Or is drizzle inextricably bound up with the life cycle of stratocumulus as is suggested in for example, Paluch and Lenschow (1991)? Influencing the life span of stratocumulus would give drizzle an important role in the climatology of stratocumulus fields and thus indirectly affect the radiative balance of the earth as well. Because it is suggested that larger aerosol concentrations negatively influence the amount of precipitation, this would lead to a direct way in which human activity modifies the climate of the earth (e.g., Albrecht 1989; Pincus and Baker 1994). To address these issues simple drizzle parameterizations are being formulated for general circulation models (e.g., Khairoutdinov and Kogan 2000; Pawlowska and Brenguier 2003). However these parameterizations are generally being implemented without a clear idea of how drizzle contributes to the existing stratocumulus climatology nor how it interacts with other processes, such as turbulent mixing and entrainment.

For these reasons we believe that a better quantification of the role of drizzle is necessary. Data collected during DYCOMS-II provide a unique opportunity to contribute to such a quantification. During DYCOMSII a downward looking 95-GHz radar (Vali et al. 1998) was mounted on the National Center for Atmospheric Research/National Science Foundation (NCAR/NSF) C130 aircraft. From this vantage point it was able to collect reflectivity data throughout the cloud layer and down close to the sea surface almost continuously during the whole time period of each flight, thus creating the possibility to get for the first time an almost continuous estimate of the surface precipitation rate over relatively large spatial scales. The results of an analysis of these data is summarized in Table 1, which shows the prevalence of drizzle during DYCOMS-II. This, and the ability to evaluate its horizontal and vertical structure using both in situ and remotely sensed data allow us to go beyond earlier studies [e.g., Austin et al. (1995) and Frisch et al. (1995), both of which had similar objectives but relied on less comprehensive instrumentation]. The remainder of this article is intended to give the reader more background on and in-depth understanding of the numbers in Table 1 . In section 2 and 3 we discuss how the rain rates of Table 1 were estimated. In section 4 we examine how $\langle R\rangle$ scales with cloud macroscopic features, at what scales drizzle is found, and the nature of its spatial and temporal variability, both within and among flights. We conclude with a discussion and a summary.

\section{The DYCOMS-II field study}

\section{a. General description}

DYCOMS-II took place in July 2001, several hundred kilometers to the west-southwest of San Diego, California. It consisted of nine flights in stratocumulustopped marine boundary layers. During the field study favorable conditions were encountered; relatively uniform, and spatially extensive stratocumulus cloud decks were probed with almost no breaks or clearings along the flight path. To a good degree of approximation the sampled boundary layers were well mixed and were capped by a strong thermal inversion; more information over the environmental conditions can be found in Stevens et al. (2003). In Fig. 1 we present satellite im-

TABLE 1. Average drizzle rates $\langle R\rangle$ (BDL denotes below detection limit) for each flight at 70-m height above the sea surface based on radar data. Each value represents roughly $5 \mathrm{~h}$ of data. The conversion from radar reflectivity to drizzle rate is done with $Z-R$ relationships derived from in situ instruments (SPP-100 and 260X) for each individual flight (RF02, RF03, RF04, RF07, RF08) or a $Z-R$ relationship derived for all nights flights (RF01 and RF05). For information on the specified uncertainty, see sections $3 \mathrm{a}$ and 5 . UTC is local time plus $7 \mathrm{~h}$.

\begin{tabular}{lcccc}
\hline \hline Flight & $\begin{array}{c}\text { Date } \\
\text { (Jul) }\end{array}$ & $\begin{array}{c}\text { Takeoff } \\
\text { (UTC) }\end{array}$ & $\begin{array}{c}\text { Landing } \\
\text { (UTC) }\end{array}$ & $\begin{array}{c}\langle R\rangle \\
\left(\mathrm{mm} \mathrm{day}^{-1}\right)\end{array}$ \\
\hline RF01 & 10 & 0601 & 1518 & BDL \\
RF02 & 11 & 0624 & 1553 & $0.35 \pm 0.11$ \\
RF03 & 13 & 0618 & 1546 & $0.05 \pm 0.03$ \\
RF04 & 17 & 0622 & 1532 & $0.08 \pm 0.06$ \\
RF05 & 18 & 0619 & 1541 & BDL \\
RF07 & 24 & 0553 & 1549 & $0.60 \pm 0.18$ \\
RF08 & 25 & 1945 & 0521 & $0.12 \pm 0.03$ \\
\hline
\end{tabular}


(a)

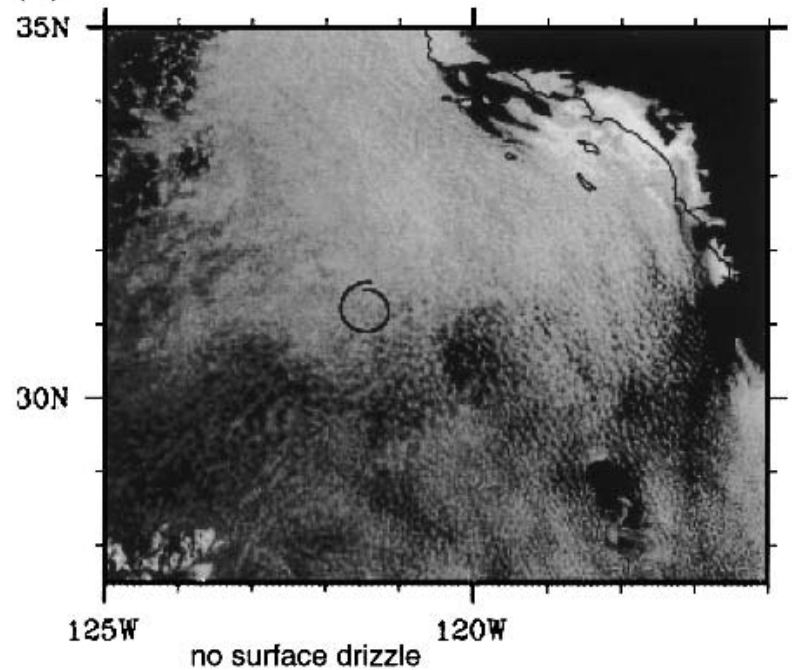

(c)

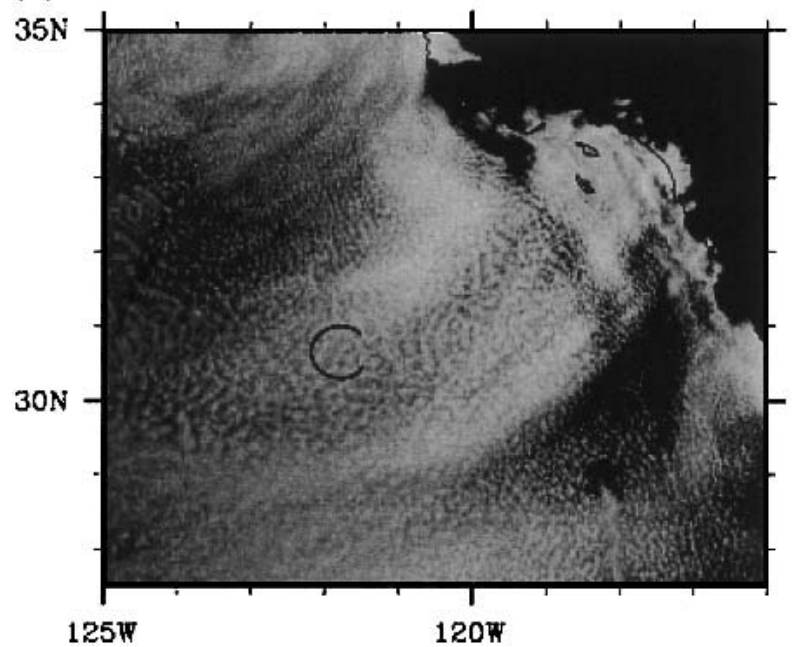

(b)

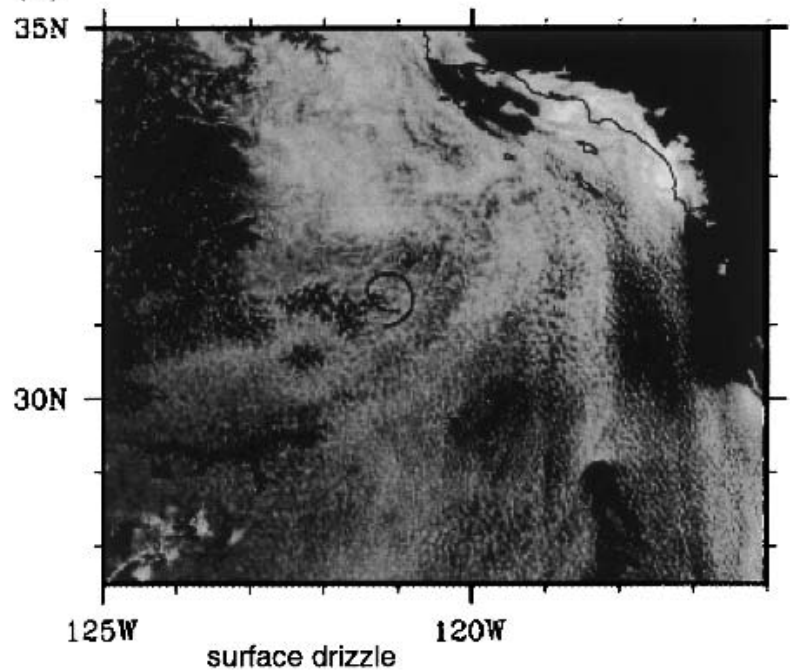

(d)

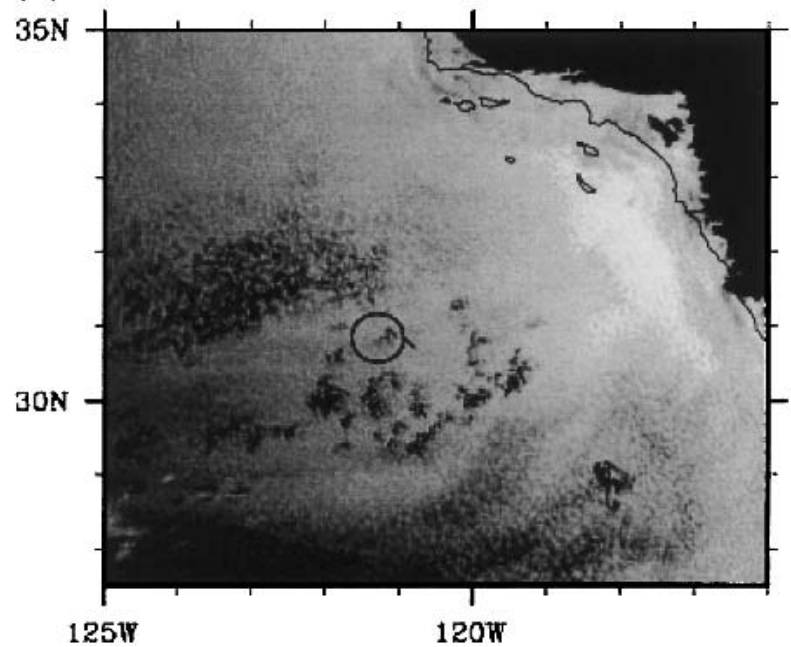

FIG. 1. Channel 1 reflectances from Geostationary Operational Environmental Satellite-10 (GOES-10) for (a) RF01, (b) RF02, (c) RF05, and (d) RF07. All pictures are first light images (1430 UTC) with the circles denoting the location of the last leg, which is more or less coincident with the time of the snapshot.

ages of four of the nine flights, two flights without drizzle reaching the surface and two flights with high values of surface drizzle rates $\langle R\rangle$. Besides showing the uniformity of the cloud layers on the scale of a measurement segment (circles of roughly $60-\mathrm{km}$ diameter) the figure also shows that the nondrizzling cases have a more uniform appearance on the large scale than the more heavily drizzling flights (e.g., Stevens et al. 2004).

One of the initial surprises of DYCOMS-II was the variability in radar-derived cloud microstructure among flights. For instance, regions of vigorous drizzle $(10 \mathrm{~mm}$ day $^{-1}$ or more) were quite common during several flights; on other flights drizzle rarely was seen below cloud base. Figure 2 encapsulates some of this variability. The differences between the structure of the nonprecipitating cloud in RF01 and its precipitating coun- terpart observed during RF07 are striking. (Note the change in scale, where $10 \mathrm{dBZ}$ corresponds to roughly an order of magnitude difference in drizzle rate.) A curtainlike echo pattern is visible during RF07, with radar reflectivities almost constant with height in places, and drizzle extending to the sea surface almost everywhere. Superimposed are local cells or pockets with significantly enhanced reflectivities, indicative of much higher drizzle rates. In contrast during RF01 radar returns are confined to the cloud layer, with only a few patches of echos extending lower down. The tendency for the reflectivity to increase with height in the cloud in this latter case is consistent with most of the radar returns coming from an adiabatic cloud microstructure, where mean particle sizes increase toward cloud top.

In addition to tantalizing data such as these, several 

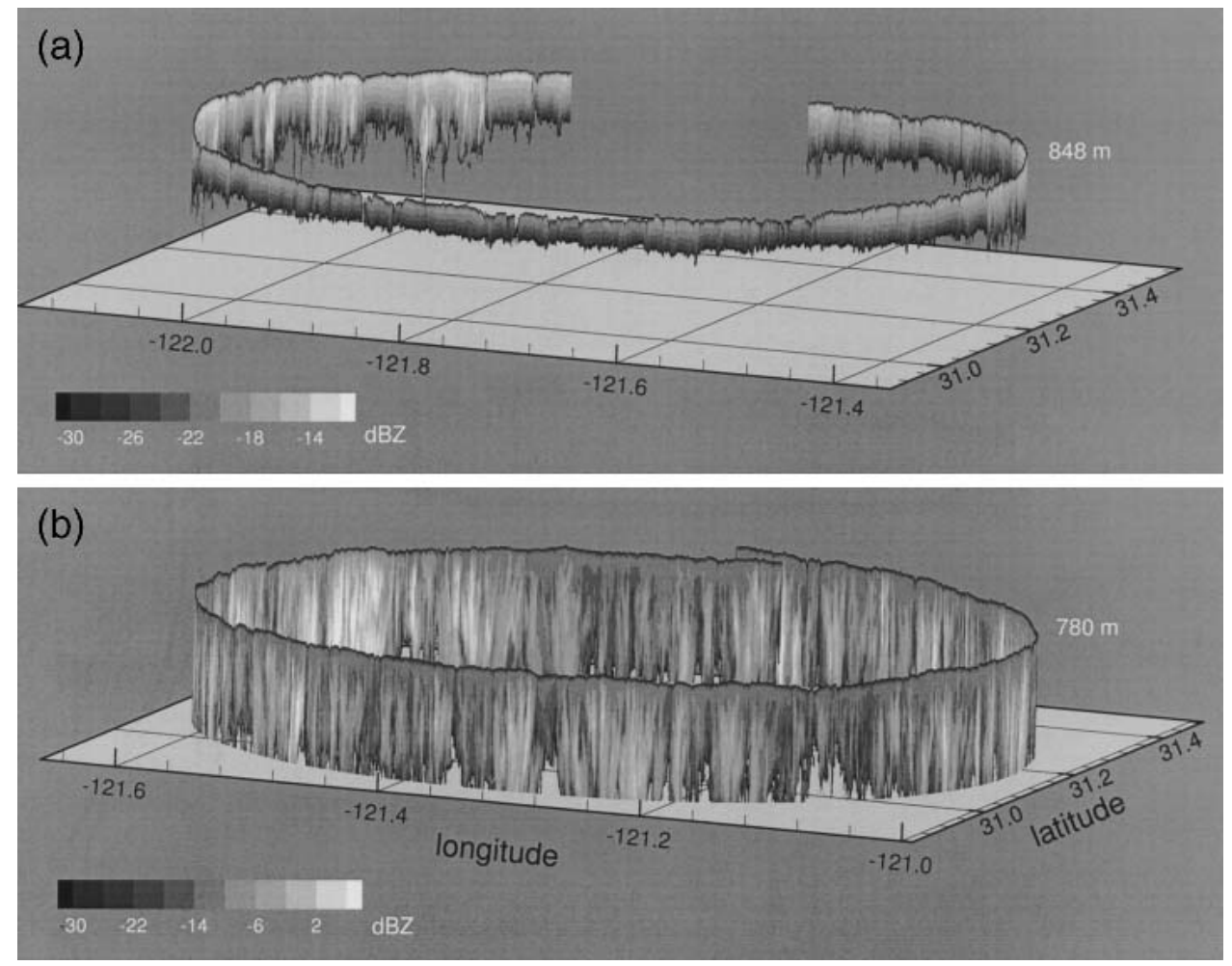

FIG. 2. Radar reflectivity of the clouds looking down from above during (a) RF01 (1146-1215 UTC) and (b) RF07 (1106-1136 UTC). Note that the scale range for both panels is different and the height specified in each panel denotes cloud top height.

technical issues also make the DYCOMS-II data appealing for further analysis. The most important being the fact that both in situ and remotely sensed data are available from which the drizzle rate can be estimated. These different types of data complement each other. Moreover, redundancy in microphysical in situ instrumentation allowed for independent estimates of, for example, the in situ drizzle rate. Secondly, all but two flights were nocturnal. Stratocumulus clouds are thought to deepen throughout the night, which is favorable for drizzle formation. Further, because the flight patterns were flown following the boundary layer wind, approximately one single air mass was probed during each flight.

Of the nine research flights flown during DYCOMSII, we focus on seven. The selected flights were chosen because they all had a similar flight pattern consisting of circles with a radius of $30 \mathrm{~km}$. The long flight legs of $\sim 30$ min enabled sufficient averaging to reduce sampling uncertainty, yet still provided insights into the spatial and temporal variability at each flight level. Usually two of the circles were flown consecutively, but in opposite directions. Within the boundary layer, measurements were concentrated at four levels; at cloud top (CT), just above cloud base (CB), just below cloud base (SC), and near the surface (SF). In addition to flight segments within the boundary layer, three remote sensing legs (RL) above the boundary layer were flown at the beginning, middle, and end of each flight. The fact that the flight strategy was almost identical for each flight facilitated intercomparison among flights. The latter was especially interesting because the flight dates and targets were specified a priori, hence the data sampled the clouds within the general target area in a manner which was not biased by preconceptions of flow patterns or statistics. The two flights not considered in this analysis were omitted because they were based on different flight patterns not conducive to this analysis. Nonetheless a qualitative examination of their structure shows them to behave consistently with the other seven flights. More detailed information about the flight plan, available instruments, specific aims of the field study, overviews of each flight, and preliminary results can be found in Stevens et al. (2003).

\section{b. Instrumentation and data quality}

The bulk of this paper is based on an analysis of measurements from a small subset of the instrumentation carried on the NCAR/NSF C130 aircraft: the 95$\mathrm{GHz}$ Wyoming Cloud Radar and three instruments for estimating the drop size distribution (DSD); one based upon single-particle scattering and the other two on shadowing of light. These last three instruments had a sample frequency of $10 \mathrm{~Hz}$. 
The SPP-100 (an electronically upgraded version of the Forward Scattering Spectrometer Probe (FSSP), SPP stands for Signal Processing Package) measured the cloud DSD (CDSD) between 2 and $47 \mu \mathrm{m}$ divided into 40 size intervals. We combined the SPP-100 data into 19 unequal-sized bins in order to minimize sizing ambiguities. During DYCOMS-II several problems were encountered with the SPP-100. For RF01 and RF02 the data are slightly questionable because it was determined that the instrument was overestimating droplet sizes by approximately two bin sizes. For the remainder of the field study another SPP-100 probe was used. This probe-sized droplets correctly; however, it failed intermittently. This introduced periods of missing data and "spikes" during restarts. We removed the spurious data points and set the data to missing value if the total number of droplet counts was zero, assuming the SPP-100 had stopped recording data. During RF05, the SPP-100 failed and no data are available for this flight. In the case of RF03 and thereafter measurements of cloud droplets were also available from a Fast-FSSP (Brenguier et al. 1998). Intercomparison of the values of the total droplet number from the SPP-100 and FFSSP for the four flights for which this was possible show that the values of the FFSSP are within $20 \%$ of the values of the SPP-100, with the SPP-100 measuring higher total droplet concentrations for all flights.

As is generally known, the liquid water amount $q_{l}$ derived from the SPP-100 is quite sensitive to the interpretation of calibration data. In the case of DYCOMSII we found an average spread of $0.1 \mathrm{~g} \mathrm{~m}^{-3}$ by comparing left- and right-handed Riemann sums (as a means of estimating the third moment $q_{l}$ ). Estimates of $q_{l}$ using centered Riemann sums tended to underestimate $q_{l}$ when compared to values as measured by bulk instruments like the Gerber Scientific, Inc. Particle Volume Monitor (PVM-100A; Gerber 1994) and the King Particle Measuring Systems probe. Compared to the PVM$100 \mathrm{~A}$ the $q_{l}$ values were $22 \%$ lower on average and compared to the King probe $12 \%$ lower. However, in at least half of the cases the PVM-100A and King probe measured values of $q_{l}$ less than what would be implied by right-handed Riemann sums. Because the difference between the SPP-100 and the King and PVM-100A probes was on the order of the difference between the third moment of the distribution as calculated by rightand left-handed Riemann sums, we simply based our analysis on the center point of a bin and accepted the error implied by our inability to determine the size of a particle within a bin.

To determine the sizes of drizzle drops, one- and two-dimensional optical array probes were used. The one-dimensional Particle Measuring Systems 260X (260X) has a theoretical range in droplet diameter from 10 to $640 \mu \mathrm{m}$ divided over 63 bins with an equal spacing of $10 \mu \mathrm{m}$; however, probe limitations combined with an aircraft speed of $100 \mathrm{~m} \mathrm{~s}^{-1}$ leads to a lower size limit of $40 \mu \mathrm{m}$ in practice. Outside of a few periods in which the
260X was nonresponsive, the probe functioned well during all flights. In particular, comparison (discussed below) with the two-dimensional optical array probe shows no discernible effect of the noise found to corrupt previous analyses (e.g., Lasher-Trapp et al. 2002). The two-dimensional optical array probe used for drizzle drops (2DC) detects particles with a diameter from $25 \mu \mathrm{m}$ up to $800 \mu \mathrm{m}$ distributed over 31 bins. The 2DC functioned properly with some exceptions; of these periods the whole of RF01 is the most noteworthy. In processing the 2DC data only particles which did not occlude either end diode were counted, this limits the size range of the probe but introduces fewer ambiguities.

The redundancy in instrumentation for the drizzle drops gives us the opportunity to compare the two. Plotting the first moment of the drizzle DSD (DDSD), as measured by both instruments, in one plot gives a first indication that, in general, the agreement between the two is quite good over a large part of the instrument's range. However, a more useful comparison is made when the fourth moment ${ }^{1}$ is used as a proxy for the rain rate. Calculation based on 120 -s averages of the correlation and regression coefficients between 100 and $500 \mu \mathrm{m}$ show that for flights RF02, RF03 (except the SF legs), RF07, and RF08 the correlation is high with values above 0.95 . Together with best-fit regression coefficients between 0.90 and 1.10 this indicates a good agreement between the $260 \mathrm{X}$ and the 2DC. For the SF legs of RF03 and flights RF04 and RF05 the agreement is poorer, with higher concentrations for the $260 \mathrm{X}$ than the 2DC for the smaller drizzle drops.

Reflectivity data were obtained with the $95-\mathrm{GHz}$ Wyoming Cloud Radar (Vali et al. 1998). The radar was operated with a dual antenna configuration, but in this paper only data from the downward-looking antenna is used. The analyzed reflectivity data had a vertical spatial resolution of $15 \mathrm{~m}$ (and sometimes $30 \mathrm{~m}$ ) and a temporal resolution of $1 \mathrm{~s}$. The radar was flown on every flight and almost continuous coverage exists with the exception of the SF legs which were too close to the surface to yield useful radar data. No attenuation corrections are applied to the data because the combination of shallow clouds with small liquid water contents yields attenuation values smaller than the $2-\mathrm{dB} Z$ calibration accuracy. The noise level of the radar displayed little variation during DYCOMS-II, so the data for all flights have been thresholded to exceed the noise level by one standard deviation based on an overall average of the recorded noise signal.

To compare the radar measurement of reflectivity $Z_{\text {radar }}$ with the integrated reflectivity estimated from the in situ probes $\left(Z_{\text {in situ }}\right), Z_{\text {in situ }}$ has been estimated by

\footnotetext{
${ }^{1}$ Drops in the drizzle drop range have fall speeds proportional to their diameter (e.g., Rogers and Yau 1989); thus, their mass flux is proportional to the fourth moment of the diameter.
} 
integrating a lognormal distribution function fitted to 120 -s averages of the data (as will be explained in section 3 ) and $Z_{\text {radar }}$ is also averaged over 120 s. As Fig. 3 shows, the correspondence between the two estimates is fairly good. Exact agreement is not to be expected because the sampling volumes of the instruments differ by several orders of magnitude and are not spatially coincident. The lack of coincidence arises because the radar during DYCOMS-II had a 140-m dead zone so that the first radar return came from volumes $\sim 150 \mathrm{~m}$ below the flight level. Further, departures from the Rayleigh scattering regime at the tail of the DSD have not been accounted for in estimating $Z_{\text {in situ. }}$. We expect this latter effect to be negligible because the maximum drizzle sizes were still considerabley smaller than the wavelength of the radar. However, an expected bias due to the height dependence of $Z$ is evident in Fig. 3. For drizzling boundary layers, such as probed during RF02, we expect $Z$ to increase from cloud top to cloud base and to decrease from cloud base to the surface (see e.g., Fig. 7c in Vali et al. 1998); thus CT points tend to be to the right of the 1:1 line and SC points tend to be to the left, indicating lower and higher $Z_{\text {in situ }}$ than $Z_{\text {radar }}$ values, respectively. Because this bias is most likely smallest for the CB legs, we calculated correlation coefficients $(r)$ and best fit regression coefficients $(s)$ for these legs. No coefficients were calculated for RF01 and RF05 because not enough data points were available to be statistically reliable. With $Z_{\text {in situ }}$ estimated from the SPP-100 and the 260X, $r$ varied between 0.73 and 0.89 and $s$ between 0.69 and 1.07 . With regard to $Z_{\text {in situ }}$ estimated from the SPP-100 and the

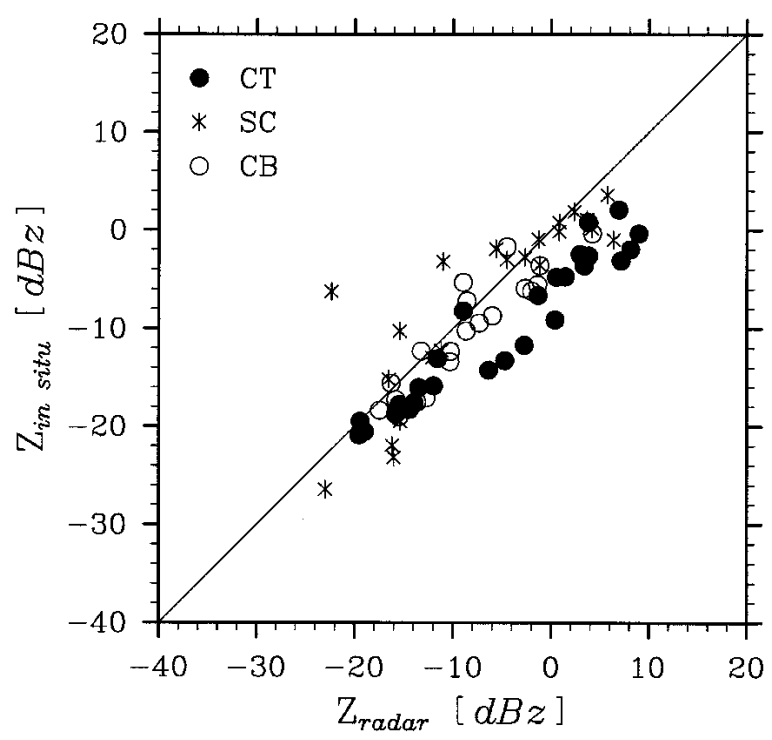

FIG. 3. Comparison of reflectivity $Z_{\text {in situ }}$ and $Z_{\text {radar }}$ with respect to RF02 for CT, CB, and SC legs; $Z_{\text {in situ }}$ is calculated from the data from the SPP-100 and the 260X. The correlation coefficient with respect to the $\mathrm{CB}$ legs data points is 0.88 and the best-fit regression coefficient is 0.82 .
2DC, $r$ varied between 0.82 and 0.97 and $s$ between 0.70 and 1.11. These numbers indicate a level of agreement which we find satisfactory but should be borne in mind when interpreting uncertainty.

\section{Analysis methods}

The in situ and remotely sensed data complement each other nicely. Computations of $R$ from the in situ data are relatively unambiguous, but can be sensitive to errors in measurements of the larger drops, which can be poorly sampled, particularly given their low and variable concentrations. The better sampling statistics of the radar help to solve this latter problem and, to the extent the ambiguity between the measured $Z$ and the desired $R$ can be resolved by the in situ data, the radar can be used to quantify precipitation over a vertical plane as opposed to along a line defined by the path of the aircraft. Although there are many objections to the use of generic $Z-R$ relations, we minimize the inherent errors by using the in situ data to tailor expressions valid on a flight to flight and level to level basis.

To do this we work entirely with in situ parametric representations of the in situ data. That is, we fit functional forms to the in situ data and then estimate the relationship between $R$ and $Z$ implied by these functional forms. The motivation for working entirely with the in situ data is that it is self-consistent and, as argued above, $Z_{\text {in situ }}$ is consistent with $Z_{\text {radar }}$, moreover $Z_{\text {radar }}$ data was not available for the surface legs. The motivation for fitting the data is that it reduces the amount of data one has to work with to a manageable level, with no apparent loss in vital information, and it provides a framework for estimating uncertainties. Further, to the extent one is confident in the fits, fitting can supply us with some extra information by extrapolating the fit to drop diameters beyond the measurement range of the instrument.

\section{a. Distribution fitting}

The choice for a specific fitting function is to a certain extent arbitrary and, although other distributions (such as the gamma function) also are attractive, we have chosen to use the lognormal one, partly because the lognormal function has been applied successfully in the past (e.g., Feingold and Levin 1986 and references herein; Gerber 1996) and partly because we were most familiar with it. Another attractive feature is that it is the expected distribution for a drizzle spectrum produced through a coalescence process dominated by long-range (in diameter or mass space) interactions (Aldous 1999). The lognormal distribution function is given by

$$
N(D)=\frac{N_{0}}{D \sqrt{2 \pi \ln ^{2} \sigma_{g}}} e^{\frac{-(\ln D-\overline{\ln D})^{2}}{2 \ln ^{2} \sigma_{g}}},
$$


with $N(D)$ the number of drops per size interval, $N_{0}$ the total number of drops, $\ln ^{2} \sigma_{g}=\overline{(\ln D-\overline{\ln D})^{2}}$, and $D$ the diameter. Note that for a lognormal distribution higher powers of a DSD are lognormally distributed as well and that distribution parameters have physical meaning: $D_{g}$ is the geometrical mean diameter or median of the size distribution given by

$$
D_{g}=e^{\overline{\ln D}},
$$

and $\sigma_{g}$ represents the geometric standard deviation or width of the distribution. Thus the fitting parameters act as proxies for the behavior of the DSDs in time and space, thereby effecting a considerable reduction in the data.

We fitted truncated lognormal functions to the observed DSDs (following Feingold and Levin 1986). This constrains the fitted distribution to have the same moments as the observed distribution over the measured size range. We fitted two lognormal distributions: one to the CDSD and the other to the DDSD. Figure 4 illustrates that this makes sense because two distinct modes are evident in the DSD, both at CT, where there are many cloud droplets present, and at SC, where still a shoulder in the DSD is readily evident. The lognormal distribution functions are fitted to DSDs averaged over two minutes (equivalent to roughly $12 \mathrm{~km}$ ). The choice for an averaging period of $2 \mathrm{~min}$ is dictated by the DDSD and is a trade-off between a longer period, which would reduce the sampling error, and a shorter period, which would allow more details to be kept in the spatial and temporal scales (see also the appendix).

In order to derive the uncertainties in the fitting parameters, an uncertainty in the DSDs has to be estimated. Because the exact instrumental error in the measurements is difficult to establish, we have chosen to take the variance in the DSD as such. A disadvantage of this is that the variance contains a large contribution (especially with respect to the larger drizzle drops) due to undersampling by the instruments. In order to overcome this drawback we reduce the output frequency of the in situ instruments by applying an intermediate averaging period of $20 \mathrm{~s}$ and define the standard deviation in the 2-min averaged DSD as the square root of the variance in those six intermediate DSDs (for more information see the appendix). Once the fit is determined, the uncertainties in the fitting parameters are calculated by first computing the $\chi^{2}$ value of the fit and next by determining how much the fitting parameters (one at a time) have to be varied in order to raise the $\chi^{2}$ value around its local minimum by 1 (Bevington and Robinson 1992). For more information see the appendix.

Before integrating the lognormal distributions to calculate $R$ and $Z$ we need to specify integration limits. For the CDSD the lower limit and upper limit of the size range of the SPP-100 are taken as $D_{\min }$ and $D_{\text {max }}$, respectively. For the DDSD $D_{\min }$ is taken equal to $D_{\max }$ of the CDSD in order to avoid either an overlap or a gap between the two distributions. For the standard calculations we chose to extrapolate the lognormal fit to $D_{\max }=1 \mathrm{~mm}$. In section 5 . sensitivity studies are presented to show the sensitivity of the choice for $D_{\max }$. More technical details about the fitting can be found in the appendix.

In Figure 5a two CDSDs measured by the SPP-100 and two DDSDs measured by the $260 \mathrm{X}$ are shown, both are 2-min averages from the first CT leg from RF07. One shows DSDs during a period of heavy drizzle $(t=23 \mathrm{~min})$ while the other is representative of a period with light drizzle (rain rates 4 times as low,
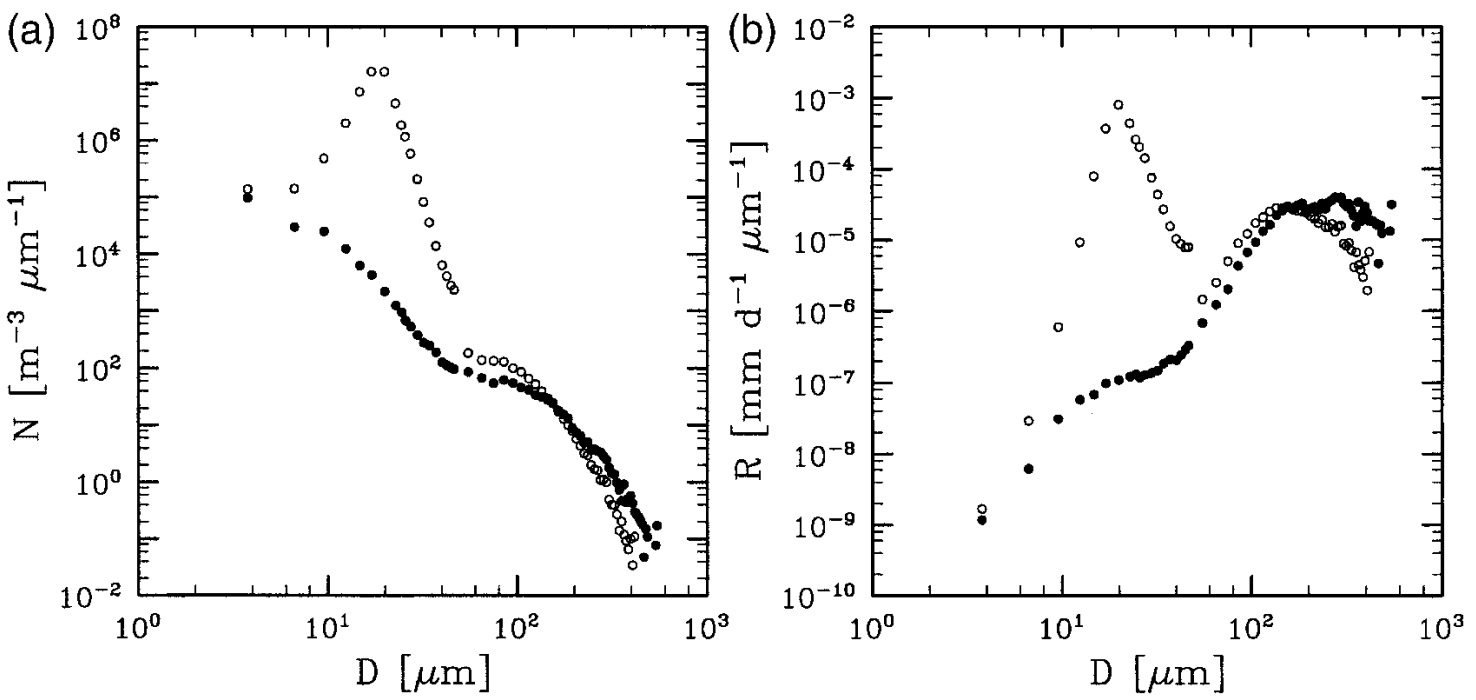

FIG. 4. (a) Leg-averaged drop concentration $N$ and (b) drizzle rate $R$ as function of the drop diameter $D$ with regard to the second CT leg (open circles) and first SC leg (closed circles) of RF07. 

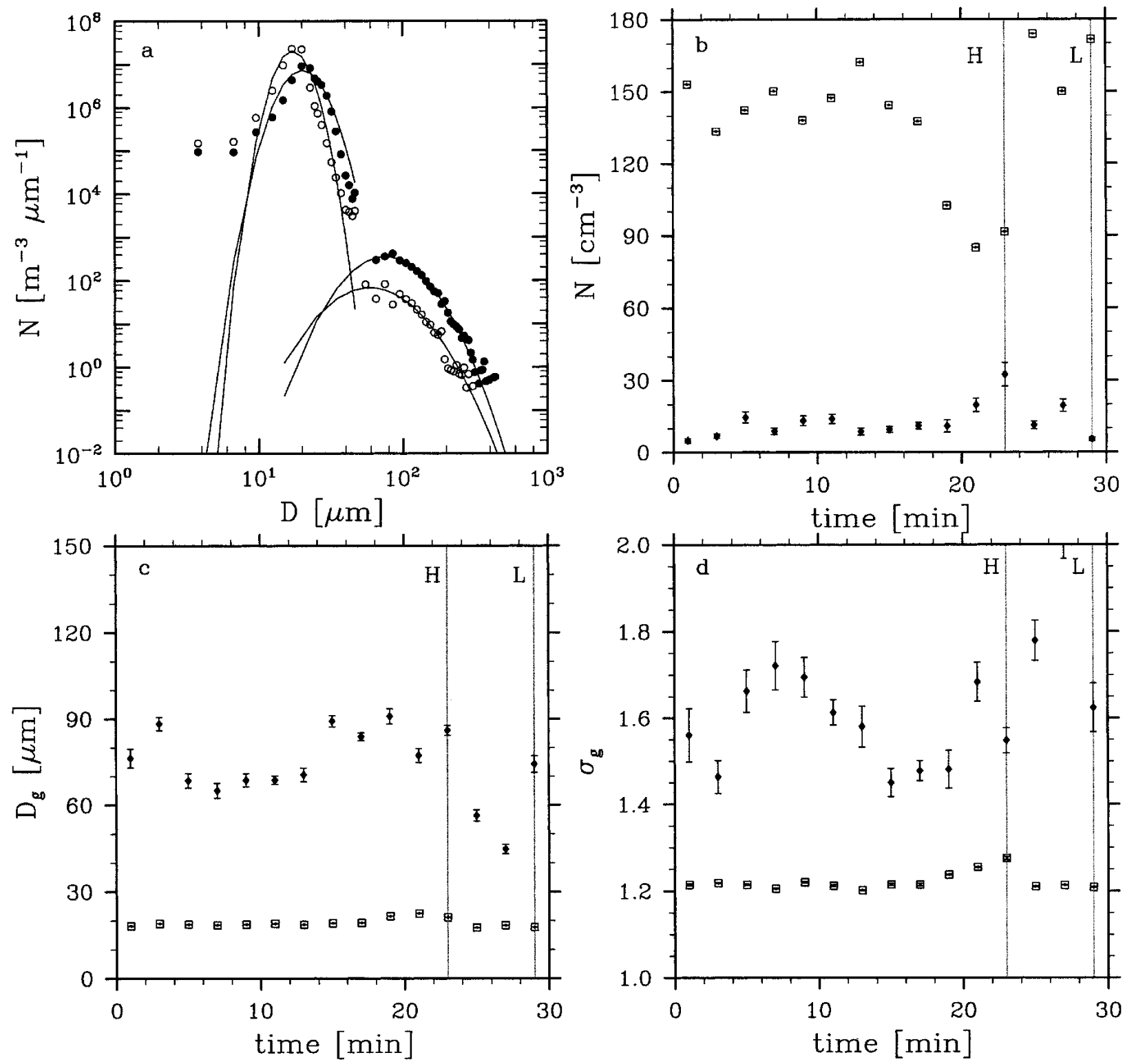

FIG. 5. Data from the first CT leg of RF07. (a) DSD of one 2-min period with heavy drizzle (closed circles) and one 2-min period with light drizzle (open circles). The functional fits to the data are given by the solid lines. For both the $260 \mathrm{X}$ (closed diamonds) and the SPP-100 (open squares) (b) drop number $N$ (note for the $260 \mathrm{X}, N$ is multiplied by 1000), (c) geometrical mean diameter $D_{g}$, and (d) geometrical standard deviation $\sigma_{g}$ as function of time. The $\mathrm{H}$ denotes the 2-min period of heavy drizzle and the $\mathrm{L}$ the period of light drizzle. Note that the error bars related to the SPP-100 fitting parameters are inside the open square symbol.

$t=29 \mathrm{~min})$. If we compare the DSDs, during the period of heavy drizzle the CDSD has a broader distribution, with a larger mean diameter and fewer droplets. The heavy drizzle DDSD shows a higher count of drops and a slightly larger mean diameter. Whether or not there is a difference in broadness is hard to judge by eye from the DSDs. The same information can be obtained, but much faster and for the whole leg, from Figs. 5b-d, which show the evolution of $N, D_{g}$, and $\sigma_{g}$ along the flight leg, both for the SPP-100 and the 260X, which suggests that the fitting parameters can be used satisfactorily as proxies for the behavior of the total DSDs.

With help of the lognormal distribution functions we analytically calculated two values for the in situ drizzle rate: one based on a combination of the 260X-SPP-100 dataset and one based on a combination of the 2DCSPP-100 dataset. A comparison between the two shows good agreement. Half of all the values of the drizzle rate based on the 260X combination are within one sigma of the drizzle rate based on the 2DC combination and $90 \%$ of the data points are within two sigma. The 
differences between the two drizzle rates are mainly due to (small) differences in the right tail end of the lognormal fits, which magnify due to our choice of extrapolating the fits up to $1 \mathrm{~mm}$. When the calculations are done up to $500 \mu \mathrm{m}$ instead, the numbers rise to around $80 \%$ and almost $100 \%$, respectively. In the rest of the article we have chosen for the sake of brevity to present in situ values for $R$ (denoted by $R_{\text {in situ }}$ ) and $Z$ (denoted by $Z_{\text {in situ }}$ ) based on data of the $260 \mathrm{X}$ and the SPP-100 only. First of all because we consider the $260 \mathrm{X}$ data to be slightly more reliable and secondly because the 2DC data had not enough acceptable 2-min fits for the SF legs to ensure a reliable $Z-R$ relationship needed for the radar reflectivity conversion to drizzle rate (see section $3 b$ ).

Because a reduced description of the DSD as collected across DYCOMS-II flights may be broadly useful, we have collected our fitting parameters into files that are available from the DYCOMS-II archive.

\section{b. $Z-R$ relationships}

An example of the rain rate reflectivity relationships that emerge from the fitted distributions during RF02 is shown in Figure 6. This figure demonstrates the extent to which a power-law relation between $R$ and $Z$ is supported by the data, it also shows that there is merit in performing the analysis at different levels. The tendency of $R$ to vary less sharply with $Z$ in the cloud is consistent with the physical expectation of the precipitation flux being carried by smaller particles (whose fall velocity is proportional to $D^{2}$ ) near cloud top and larger particles (whose fall velocity is proportional to $D$ ) lower down. Although we show above $Z-R$ relations for each flight and for each flight level, in the remainder

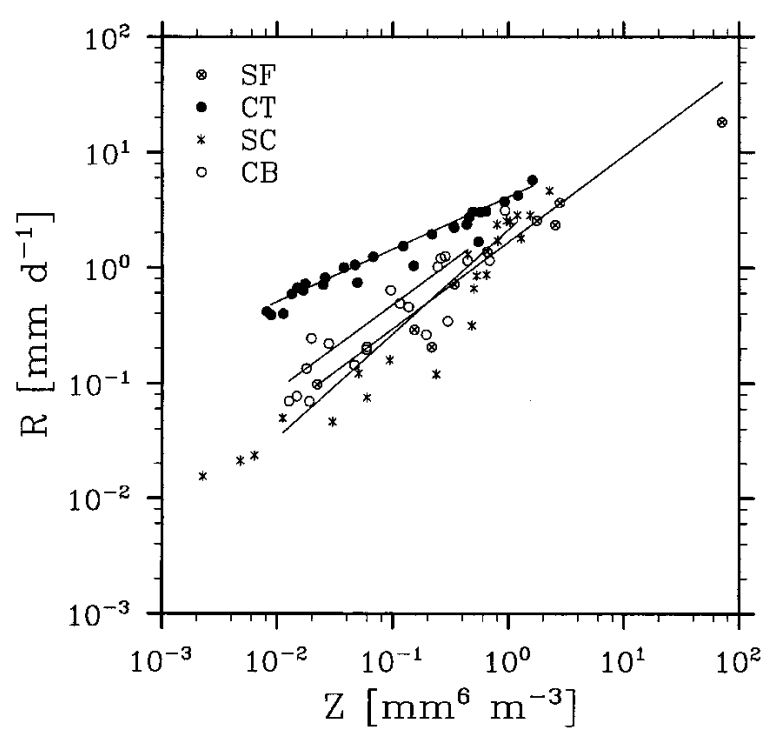

FIG. 6. Drizzle rate $R_{\text {in situ }}$ vs reflectivity $Z_{\text {in situ }}$ for RF02 at four different leg heights: CT, CB, SC, and SF. Best fits are also given based on the two legs flown at each height. of the manuscript we focus on two levels: one corresponding to the height of the SF legs, which we call the surface, and one at the height of the CB legs, which we call cloud base.

More generally, in Table 2 we present $Z-R$ relations valid at cloud base and at the surface for each flight for which significant drizzle was evident. The uncertainties in these relations are estimated by propagating the uncertainties in our fits of the distributions. The relationships in Table 2 will be used in section 4 to compute drizzle rates from the radar reflectivities. To avoid ambiguity with in situ drizzle rates, those calculated from the radar reflectivity will be denoted by $R_{\text {radar }}$.

\section{Variability of drizzle}

Keeping in mind that an $R$ of $1 \mathrm{~mm} \mathrm{day}^{-1}$ is roughly equivalent to a heat flux of $30 \mathrm{~W} \mathrm{~m}^{-2}$ (which is in general comparable to half the net longwave radiative flux divergence at cloud top), the flight-averaged drizzle rate $\langle R\rangle$ in Table 1 gives a first impression of the importance of drizzle for the overall energetics of the PBL. Based on this, the seven flights naturally divide into three groups. RF02 and RF07 can be characterized as "heavy" drizzle cases, while in flights 3,4 , and 8 only a modest amount of drizzle reached the surface. Both RF01 and RF05 belong to the "very light" or "no drizzle" group because only trace amounts of drizzle reached the surface. Incidentally a qualitative analysis of radar echoes from RF06 and RF09 put them in the heavy and very light drizzle categories, respectively.

Those data support the idea of an existing diurnal cycle in the drizzle rate (i.e., higher drizzle rates during the night compared to lower daytime values), especially if RF06 (nighttime flight) and RF09 (daytime flight) are taken into account as well. However, the experimental strategy did not allow us to detect an early morning maximum in the drizzle rate (Kraus 1963).

In the analyses we define drizzle as having a drizzle rate of at least $0.03 \mathrm{~mm}$ day $^{-1}$ to avoid different minimum $R_{\text {radar }}$ thresholds for every leg. (Note that this

TABLE 2. Values for parameter $a$ and power $n$ in the $Z-R$ relationship $R=a Z^{n}$ based upon the data of the SPP-100 and $260 \mathrm{X}$, both of the SF legs and the CB legs. For RF01 and RF05 not enough data points are available from the in situ data so in these cases, $R$ was related to $Z$ using a relationship derived from the average of either all the nocturnal flights, or all of the flights. These relationships are used to compute drizzle rates from radar reflectivities at $70 \mathrm{~m}$ and mean cloud-base height, respectively.

\begin{tabular}{lccccc}
\hline \hline & \multicolumn{2}{c}{ SF } & & \multicolumn{2}{c}{ CB } \\
\cline { 2 - 3 } \cline { 5 - 6 } Flight & $a$ & $n$ & & $a$ & $n$ \\
\hline RF02 & $1.66 \pm 0.27$ & $0.75 \pm 0.13$ & & $2.60 \pm 0.25$ & $0.74 \pm 0.02$ \\
RF03 & $0.94 \pm 0.38$ & $0.62 \pm 0.15$ & & $1.82 \pm 0.19$ & $0.61 \pm 0.03$ \\
RF04 & $0.86 \pm 0.49$ & $0.58 \pm 0.18$ & & $1.66 \pm 0.12$ & $0.59 \pm 0.04$ \\
RF07 & $1.12 \pm 0.23$ & $0.66 \pm 0.13$ & & $2.13 \pm 0.10$ & $0.68 \pm 0.04$ \\
RF08 & $1.22 \pm 0.24$ & $0.47 \pm 0.04$ & & $2.68 \pm 0.10$ & $0.46 \pm 0.01$ \\
Night & $1.31 \pm 0.14$ & $0.74 \pm 0.04$ & & $2.05 \pm 0.07$ & $0.67 \pm 0.01$ \\
All & $0.51 \pm 0.03$ & $0.34 \pm 0.02$ & & $2.73 \pm 0.07$ & $0.68 \pm 0.01$ \\
\hline
\end{tabular}


lower limit is equivalent to the removal of one liter water per day over an area of $5 \mathrm{~m} \times 6 \mathrm{~m}$.) Hereafter, we will refer to drizzle rates of $1 \mathrm{~mm} \mathrm{day}^{-1}$ and higher as heavy drizzle.

\section{a. Interflight variability}

While most physically based investigations have rightfully focused their attention on physical interactions, less attention has been devoted to the question of the statistics of drizzle as a function of cloud macroscopic properties. It seems worthwhile to take a more empirical approach and ask whether in spite of such complexities observed drizzle rates covary in some simple way with cloud macrophysical properties. Such an approach is motivated by the realization that many simple microphysical models produce such scaling in their stationary limit (e.g., Pincus and Baker 1994), and recent observational work suggests that the cloudaveraged drizzle flux can be expressed as a power law of cloud depth and cloud droplet concentration in adiabatic regions of the cloud layer (Pawlowska and Brenguier 2003). In addition to providing a target for future theoretical work, such relationships (insofar as they exist) can form the basis for parameterizations of drizzle in large-scale models and also aid retrievals of drizzle from satellite-derived estimates of cloud macroscopic properties.

To begin, we follow the lead of Pawlowska and Brenguier (2003) and ask to what extent the drizzle rate at cloud base scales with cloud depth $H$ and cloud droplet concentration $N$. In this analysis we estimate $H$ using the data tabulated in Stevens et al. (2003) (for easy reference $\left\langle h_{\mathrm{ct}}\right\rangle$ is included in Table 3$)$, which corresponds to the difference between the flight-averaged cloud-top height, $\left\langle h_{\mathrm{ct}}\right\rangle$, and the flight-averaged cloud base height, $\left\langle h_{\mathrm{cb}}\right\rangle$. The former is derived from lidar measurements of cloud top made during the three RL legs (roughly $90 \mathrm{~min}$ of $1 \mathrm{~s}^{-1}$ data). The latter is based on roughly $4 \mathrm{~h}$ (per flight) of in situ data collected from flight legs flown in or below the cloud layer. Variability in $h_{\mathrm{ct}}$ and $h_{\mathrm{cb}}$ was typically $20-50 \mathrm{~m}$, although in RF04 and to a lesser extent in RF05 there is evidence of an almost discrete change in cloud top and base indicative of sampling across two distinct air masses. To estimate $N$ we average the SPP-100 data from all the cloud legs, which typically corresponds to $2 \mathrm{~h}$ of data. Because $N$

TABLE 3. Macroscopic variations in cloud structure and rain rates among flights. See text for definitions.

\begin{tabular}{lccccc}
\hline \hline Flight & $\begin{array}{c}H \\
(\mathrm{~m})\end{array}$ & $\begin{array}{c}\left\langle h_{c t}\right\rangle \\
(\mathrm{m})\end{array}$ & $\begin{array}{c}N \\
\left(\mathrm{~cm}^{-3}\right)\end{array}$ & $\begin{array}{c}\Delta T \\
(\mathrm{~K})\end{array}$ & $\begin{array}{c}R_{\text {radar,cb }} \\
\left(\mathrm{mm} \mathrm{day}^{-1}\right)\end{array}$ \\
\hline RF01 & 265 & 850 & 140 & $\mathrm{n} / \mathrm{a}$ & 0.05 \\
RF02 & 360 & 800 & 58 & 1.6 & $1.29 \pm 0.14$ \\
RF03 & 390 & 700 & 254 & 3.3 & $0.18 \pm 0.02$ \\
RF04 & 465 & 1075 & 205 & 2.2 & $0.76 \pm 0.07$ \\
RF05 & 275 & 925 & 151 & 3.1 & 0.04 \\
RF07 & 515 & 825 & 135 & 1.9 & $1.65 \pm 0.13$ \\
RF08 & 330 & 600 & 113 & 2.3 & $0.38 \pm 0.02$ \\
\hline
\end{tabular}

tends to vary relatively little through the depth of the cloud, such an average seems warranted. For the radarderived drizzle rate at flight-averaged cloud base $R_{\text {radar,cb }}$ we use reflectivity time series from the CT and RL legs, which corresponds to, on average, $150 \mathrm{~min}$ of data per flight.

Values of $H, N$, and $R_{\text {radar,cb }}$ calculated in the above described manner are given in Table 3. As illustrated in Fig. 7a these data seem to support an $R_{\text {radar.cb }} \propto H^{3} / N$ relationship. This finding differs from the $H^{4} / N$ scaling that Pawlowska and Brenguier found for clouds sampled during the second Aerosol Characterization Experiment (ACE-2). However, their drizzle rates are being reevaluated to account for new information about the sample volume used in the probes (J. L. Brenguier 2004, personal communication).

Past studies have also attempted to relate drizzle to satellite-based estimates of particle size, such as the cloud-top effective radius $r_{e}$. For instance, using ground and satellite-based remote sensing Han et al. (1995) argue that drizzle could be associated with occurrences of satellite-derived estimates of $r_{e}>15 \mu \mathrm{m}$, and that clouds could be categorized as nonprecipitating when $r_{e}<10 \mu \mathrm{m}$. Using only in situ data Gerber (1996) presents evidence that, whenever $r_{e}$ exceeds a $16-\mu \mathrm{m}$ threshold, drizzle tends to be heavy. He argues that this suggests the presence of a coalescence threshold, (e.g., Hocking 1959). Ship-track data analyzed by Ferek et al. (2000) also show evidence of a thresholdlike dependence of drizzle on cloud-top effective radius, with a threshold lying somewhere between 9 and $14 \mu \mathrm{m}$. Note that the somewhat more fuzzy threshold behavior in the Han et al. and Ferek et al. studies relative to the measurements of Gerber may in part be due to the different nature of the sampling. Gerber's measurements essentially show a discreet change in the structure of the local droplet spectrum as its effective radius increases beyond a certain value. The other studies speak more to the aggregate properties of precipitating versus nonprecipitating cloud layers. For the purposes of this study we are more interested in the latter.

To address the question of a possible relationship between drizzle rate and $r_{e}$ we compare $R_{\text {radar,cb }}$ with $r_{e}$ estimated using in situ data collected along the cloud top legs. For these purposes we estimated $r_{e}$ from the fits to the SPP-100 and 260X data. Results from all analyzed flights are plotted in Fig. 8. Overall they support the idea of thresholdlike behavior between 10 and $15 \mu \mathrm{m}$. However the transition between large and small values of $R_{\text {radar,cb }}$ is not particularly sharp and there is evidence of systematic differences among flights. Because for an adiabatic cloud $r_{e}$ scales with $H^{1 / 3}$, this is not likely to be due to systematic differences in relative distance from cloud top among flights (and hence biases in our estimate of $r_{e}$ ).

Finally we compare drizzle rates to the difference $\Delta T$ between the $11-\mu \mathrm{m}$ and $4-\mu \mathrm{m}$ brightness temperatures as measured by GOES-10. This is instructive be- 

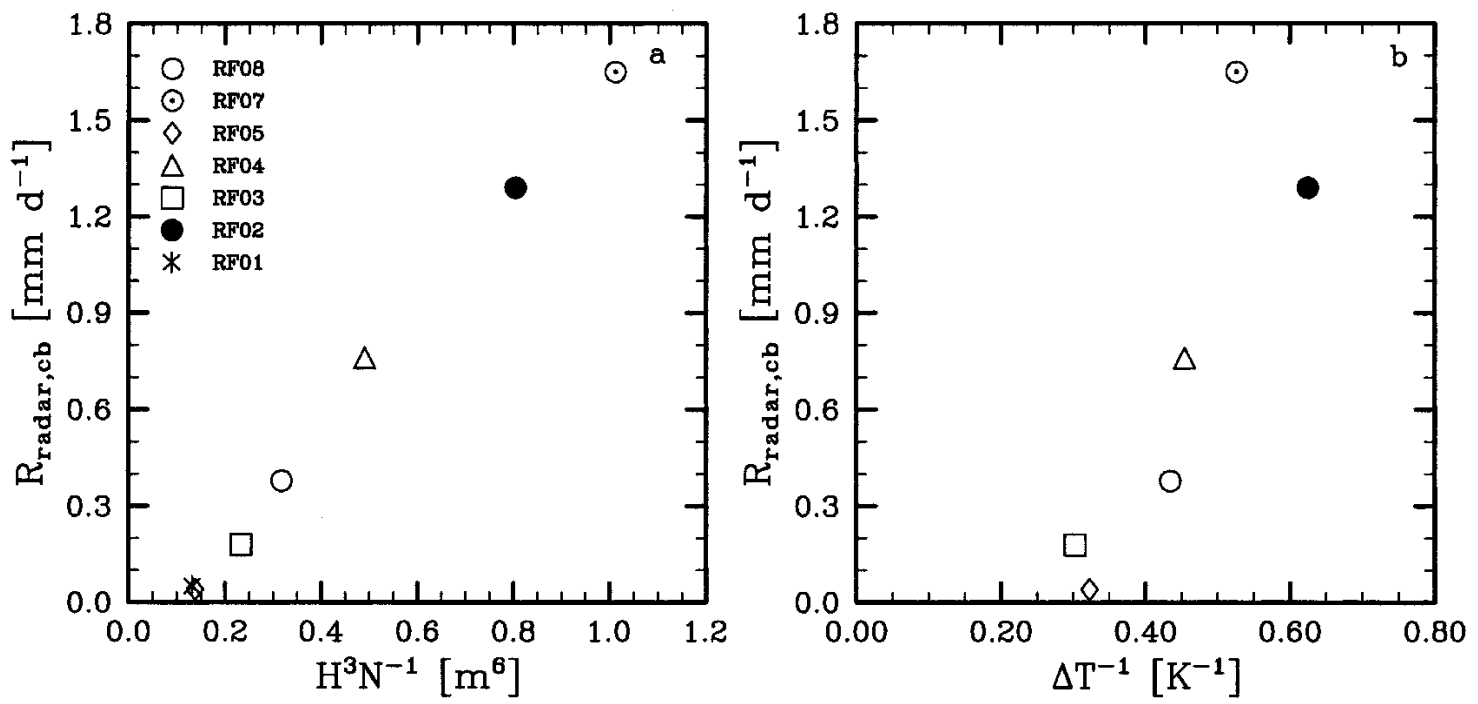

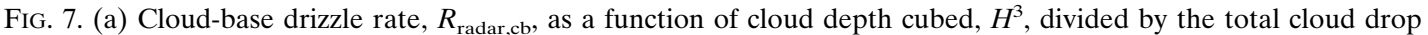
number $N$. (b) Cloud-base drizzle rate vs the inverse of $\Delta T$.

cause the comparison between the drizzle rate and $r_{e}$ as derived from satellite measurements are made more difficult by the lack of standard nocturnal retrievals for $r_{e}$ and the tendency of the daytime retrievals to fail in regions where the cloud becomes more broken. Because drizzle seems to correlate with more broken clouds (see Fig. 1) such a failure might significantly bias the measurements. Using $\Delta T$ instead is feasible because, for example, for a cloud with an optical depth of 15 , changes in $r_{e}$ from 6 to $12 \mu \mathrm{m}$ will result in a decrease of $\Delta T$ from approximately $5 \mathrm{~K}$ to nearly $1 \mathrm{~K}$. In contrast, such changes at a fixed value of $r_{e}$ would require a 15 -fold reduction in the optical depth (cf. Fig. 1 of Perez et al. 2000). All flights except RF08 were nocturnal, so $\Delta T$ values were estimated using the 1200 UTC GOES-10 image, while for RF08 the 0300 UTC image was used. Using $\Delta T^{-1}$ as a proxy for drop size, Table 3 shows a clear tendency for bigger drops to be associated with fewer drops and more drizzle. The latter correlation is depicted in Fig. 7b. RF06 and RF09, whose flight patterns were not conducive to the quantification of drizzle, also fit this pattern. By using 0.25 $\mathrm{K}<\Delta T<2 \mathrm{~K}$ as a proxy for regions where $R>1 \mathrm{~mm}$ day $^{-1}$ then the nighttime imagery can be exploited to estimate drizzle rates over larger areas. In the 1200 UTC GOES-10 nighttime imagery, the box bounded by $35^{\circ}-30^{\circ} \mathrm{N}, 125^{\circ}-120^{\circ} \mathrm{W}$ has $R>1 \mathrm{~mm}^{-1 a y}{ }^{-1}$ in overcast regions $27 \%$ of the time. This proportion of drizzle is consistent with the fact that approximately one-third of the DYCOMS flights measured significant to heavy drizzle at cloud base.

\section{b. Horizontal variability}

Figures 9 and 10 show the spatial distribution of drizzle for flights RF02 and RF07, the two nighttime flights with the highest $\langle R\rangle$. The precipitation rate at flight level can be read by subtracting the baseline height and associating $100-\mathrm{m}$ increments with $1 \mathrm{~mm}$ day $^{-1}$. For both flights we show data from the two SC and CT legs since they form a representative cross section of the whole flight and were flown consecutively (except for the case of RF07 where the CT legs were interrupted by the remote sensing leg whose radar echos are shown in Fig. 2). The averaged precipitation rate during the four legs shown is actually equal for both flights $\left(0.5 \mathrm{~mm} \mathrm{day}^{-1}\right)$. Yet $\langle R\rangle$ is roughly twice as high for RF07 compared to RF02; this is due to drizzle reaching the surface during the whole span of RF07,

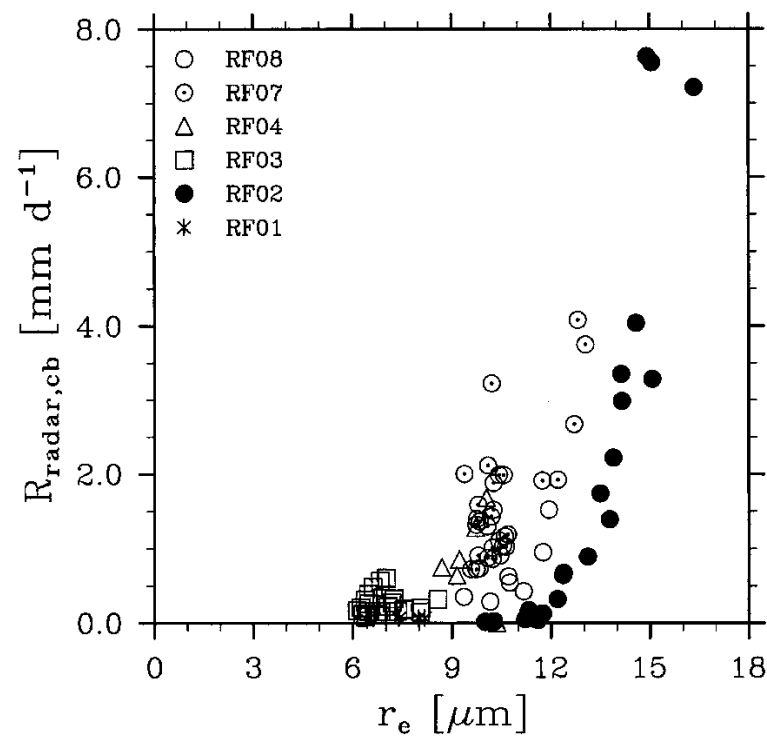

FIG. 8. Cloud-base drizzle rate, $R_{\text {radar.cb, }}$ vs the effective radius $r_{e}$ at cloud top for all flights except RF05. Each data point represents 2 min of data. 
(a)

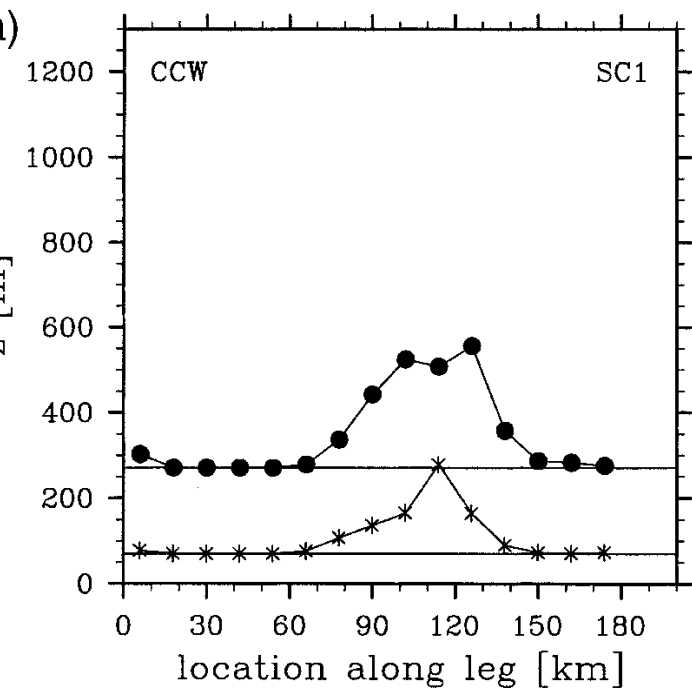

(c)

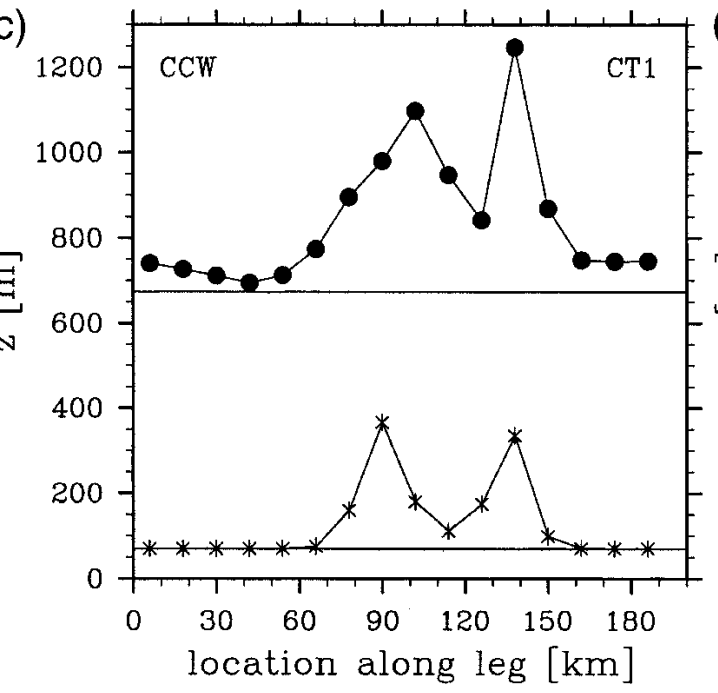

(b)

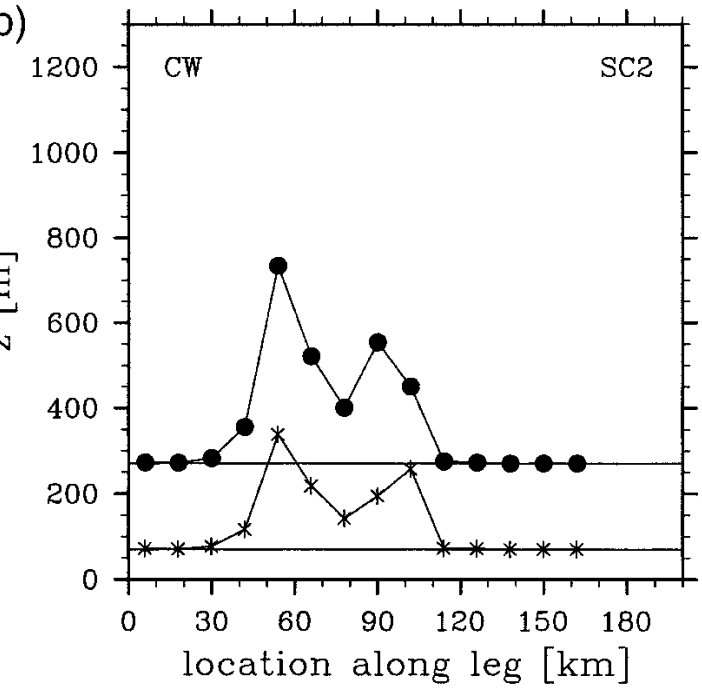

(d)

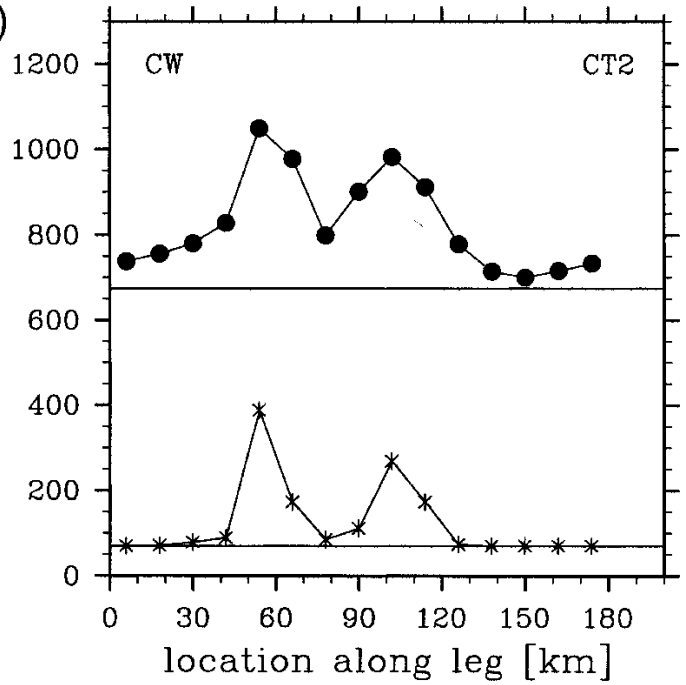

Fig. 9. Drizzle rate $R$ during RF02 for the two SC and CT legs as function of location along the flight leg. Here $R_{\text {in situ }}$ (closed circles) and $R_{\mathrm{radar}}$ (asterisks) are plotted relative to a baseline denoting the measurement level, with $1 \mathrm{~mm}^{\mathrm{iny}}{ }^{-1}$ corresponding to $100 \mathrm{~m}$. The flight direction [counterclockwise $(\mathrm{CCW})$ and clockwise $(\mathrm{CW})$ ] is specified in the upper-left corner of each panel.

while during RF02 surface precipitation was very low in the beginning.

It is reassuring to see the similarity in spatial structure (on the order of $10-\mathrm{km}$ scale) between the in situ data and surface radar data. To a certain extent this is expected but on the other hand several factors could have contributed to differences between the two. Drizzle measured at a cloud base of $500 \mathrm{~m}$ could be expected to reach the surface nearly 30 min later (i.e., assuming a mode diameter of $100 \mu \mathrm{m}$, which corresponds to a fall speed of $0.3 \mathrm{~cm} \mathrm{~s}^{-1}$ ); hence, if the time scale of drizzle evolution is much shorter than this, we would anticipate little coherence in the vertical. The degree of vertical coherence observed is consistent with the apparent temporal coherence, as evident in the persistence of the envelope of precipitation among two or even more legs. (Keep in mind that consecutive legs are flown in opposite directions, thus $R$ in the different panels displays a mirror symmetry.) Other indications of a time scale for drizzling regions of at least an hour and potentially much longer can be found when radar echo images of consecutive legs are studies by eye: a clear persistence on larger scales is quite often visible.

An apparent difference between RF02 and RF07 is the higher background drizzle rate of RF07. The large rain rates observed during RF02 appear to be localized into small regions or cells. The contribution of such cells to the overall drizzle rate of RF07 seems less pronounced. The net contribution of regions of varying precipitation rate to the observed accumulation is perhaps better illustrated in Fig. 11. Figure 11a presents normalized distributions of $R_{\text {radar }}$ for RF02, RF07, and 
(a)

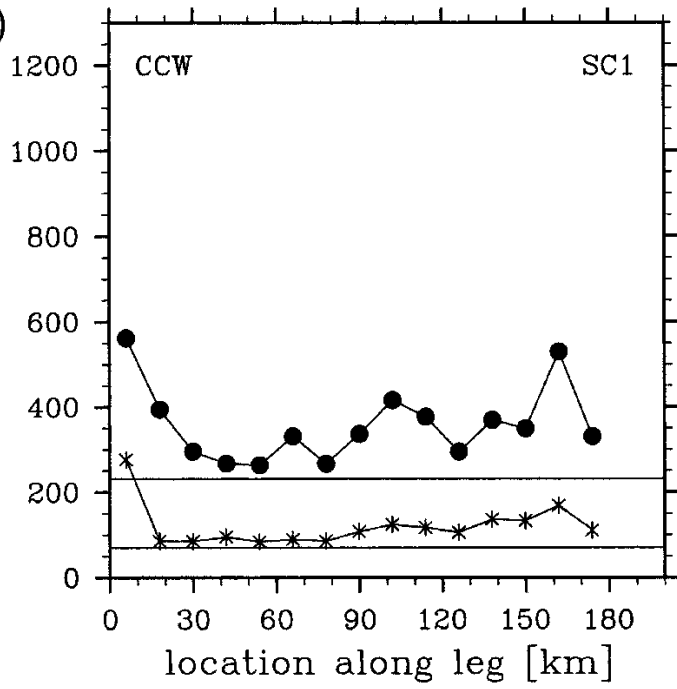

(c)

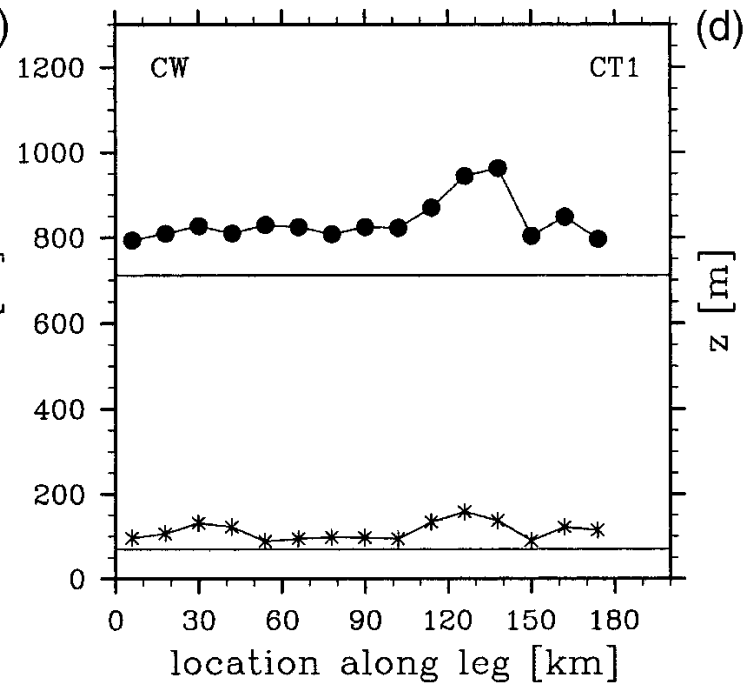

(b)

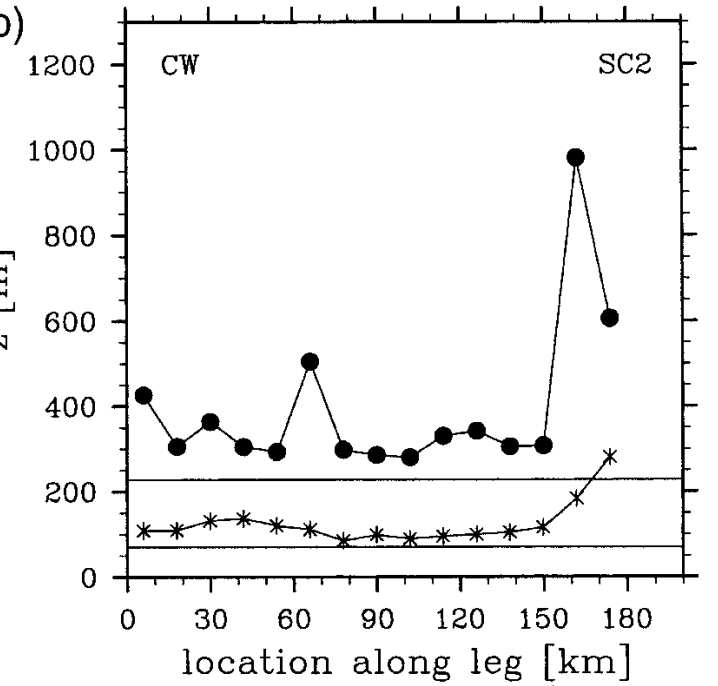

d)

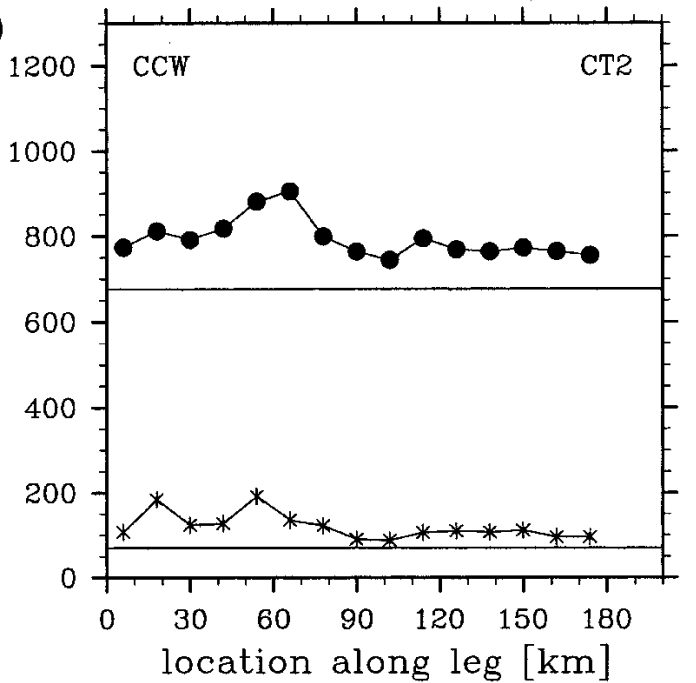

FIG. 10. As in Fig. 9 but for RF07.

RF04; RF03 and RF08 behave similar to RF04 and are omitted for clarity. The drizzle rate on the vertical axis denotes the amount of drizzle in the bin interval as a fraction of $\langle R\rangle$ (Table 1). Note that the bins on the horizontal axis are logarithmic. Figure 11b shows the cumulative distribution of $R_{\text {radar }}$ scaled with $\langle R\rangle$ as a function of the fraction of the total drizzling area. The percentage of the total flight pathlength with drizzle at the surface can be obtained from Table 4.

The visual similarity among the three distributions in Fig. 11a is supported by a more quantitative analysis, suggesting that the distribution of drizzle intensity could be captured by a simple parametric representation. However because of the logarithmic abscissa, a rightward shift of the distribution sharply increases the extent to which the relatively rare, but intense drizzle events contribute to the overall distribution. This is evident in Fig. 11b, which shows that in the case of RF02 only $20 \%$ of the drizzling area is responsible for $80 \%$ of the total amount of drizzle removed from the boundary layer. Further insight into these issues is provided in Table 4, which examines how frequently drizzle reaches the surface on a given flight and what fraction of the drizzle can be considered heavy. Besides showing once more the importance of the heavy drizzling cores to the overall drizzle rate, the table also shows that low overall drizzle rates correlate with a low intensity of drizzle. Interestingly this implies that the greater the value of $\langle R\rangle$, the more likely it is that drizzle (of a significant amount) covers small spatial areas - consistent with the idea that drizzle could induce a transition in cloud structure (Stevens et al. 1998; Paluch and Lenschow 1991).

\section{c. Dependence on droplet spectra}

Even though it is to be expected from first principles, and clearly supported by Fig. 7, measurements are not available to examine the influence of local cloud depth on local drizzle rates. Although cloud top can be de- 

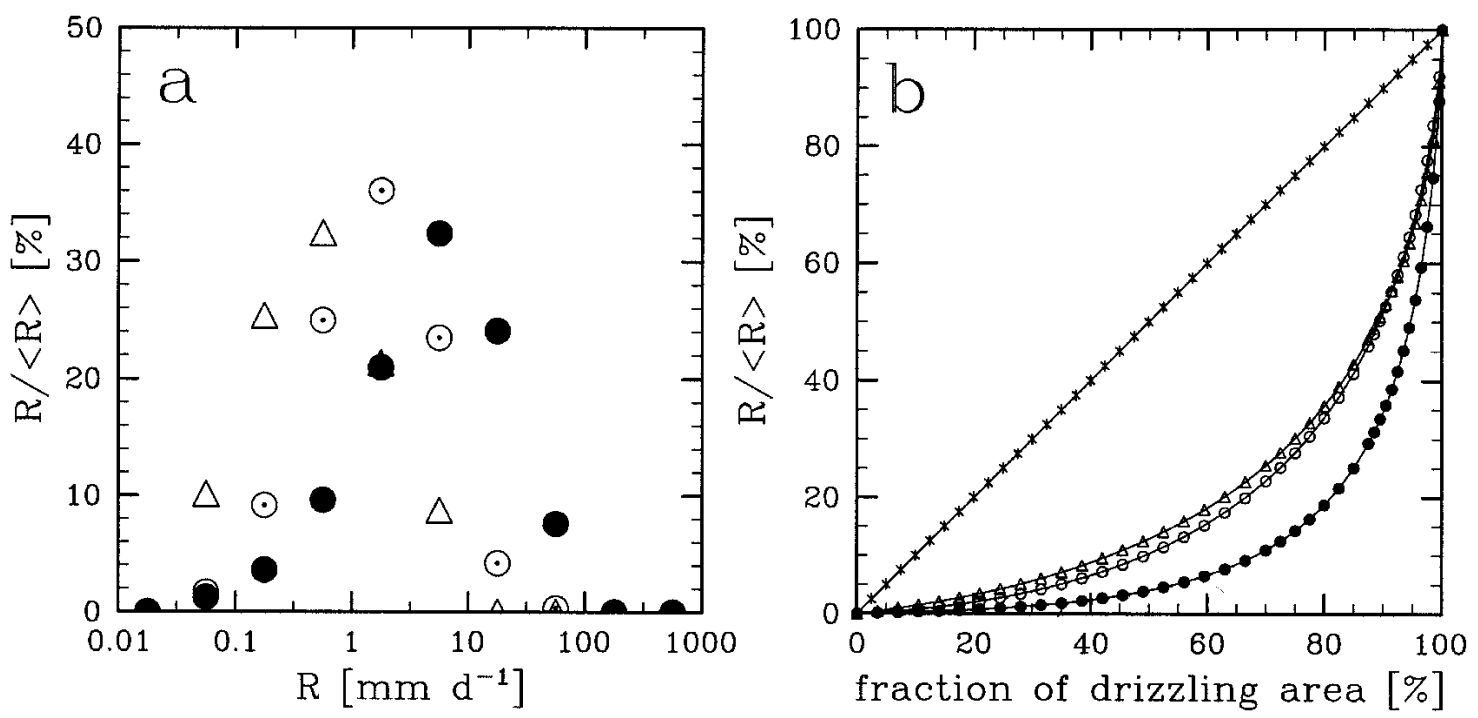

FIG. 11. (a) Distribution of the drizzle-rate intensity for RF02, RF07, and RF04 as function of the relative contribution to $\langle R\rangle$, and (b) the cumulative distribution of $R$ scaled with $\langle R\rangle$ as function of the fraction of the total drizzling area. Symbol representation similar to Fig. 7. The straight line depicts a uniform distribution.

tected when flying above the cloud and looking down, the radar does not well represent cloud base. Similarly, when flying below the cloud the local lifting condensation level can be used as a proxy for cloud base, but in this case no cloud top information is available. On the other hand, we can gain some insight by examining the correlations between $\langle R\rangle$ and the parameters of the fitted droplet distributions. Figure 12 shows that the best correlation is found between the number of drizzle drops and $R_{\text {in situ }}$. This indicates that increased drizzle is not accompanied by a change in shape of the part of the droplet distribution associated with drizzle. This suggests that it might suffice to model the drizzle mode using a one-parameter distribution. There is also evidence of a weaker negative correlation between $R_{\text {in situ }}$ and the number of cloud droplets. Together with a (nearly) constant $D_{g}$, both for the cloud and the drizzle drops, and a slight broadening of the CDSD, this is consistent with the scavenging of cloud droplets by precipitation. For another example see Fig. 5b. Signs of precipitation scavenging were also noticed by Austin et al. (1995), e.g., their Fig. 7.

Using the fact that $D_{g}$ and $\sigma_{g}$ appear to be (nearly) constant over a leg, we estimate how much of the variability in $R_{\text {in situ }}$ can be associated with variability in $N$. To do this, in Fig. 12a we compare $R_{N}$ with $R_{\text {in situ, }}$, where $R_{N}$ is the value $R_{\text {in situ }}$ would have if $D_{g}$ and $\sigma_{g}$ were fixed at their mean values. Here $R_{N}$ captures the variability of $R$ very well and no systematic bias is evident. This is also demonstrated by the fact that $\bar{R}$ and $\overline{R_{N}}$ (with the overbar denoting leg-averaged values of $R_{\text {in situ }}$ and $R_{N}$ ) are so close that the two lines indicating those values in the figure are indistinguishable.

Figure 13 reveals that similar results are valid for other legs as well. The figure presents $\bar{R}$ versus $\overline{R_{N}}$ for all seven flights. The correlation between the two is high with $r$ of 0.95 and the data does not deviate a lot from a one-to-one line. Figure 12 is somewhat misleading in suggesting that variability in $D_{g}$ and $\sigma_{\mathrm{g}}$ is unimportant; however, comparison of $\bar{R}$ with $\overline{R_{D_{g}}}$ and $\overline{R_{\sigma_{g}}}$ (calculated with local values of $D_{g}$ and $\sigma_{\mathrm{g}}$, respectively and leg-averaged values of the other two fitting parameters) also displays correlation coefficients with acceptable values. With respect to $\bar{R}$ and $\overline{R_{D}}, r$ has a value of 0.73 and for $\bar{R}$ and $\overline{R_{\sigma_{g}}} r$ is 0.78 , thus reflecting the lack of independency among the fitting parameters (Haddad et al. 1996). Yet, an interesting side effect of $R_{N}$ explaining most of the variability in $R_{\text {in situ }}$ is the fact that a drizzle rate calculated with leg-averaged values for all three fitting parameters (thus representing a legaveraged $R_{N}$ ) has the same high $r$ of 0.95 due to the fact that the calculation of the drizzle rate is linear in $N$.

\section{d. Subcloud evaporation}

To investigate evaporation in the subcloud layer we calculate the fraction of the cloud-base drizzle rate that

TABLE 4. Values for each flight of the percentage of time or space that drizzle is detected at $70 \mathrm{~m}$ above the sea surface, that heavy drizzle $\left(R>1 \mathrm{~mm} \mathrm{day}^{-1}\right)$ is detected, and the contributions of heavy drizzle to the total drizzle rate.

\begin{tabular}{lccc}
\hline \hline Flight & $\begin{array}{c}L_{R} / L_{\text {tot }} \\
(\%)\end{array}$ & $\begin{array}{c}L_{R_{\text {heavy }}} / L_{\text {tot }} \\
(\%)\end{array}$ & $\begin{array}{c}R_{\text {heavy }} / R_{\text {tot }} \\
(\%)\end{array}$ \\
\hline RF01 & 0.1 & 0.0 & 0.0 \\
RF02 & 27.0 & 6.6 & 85.4 \\
RF03 & 27.7 & 0.6 & 19.7 \\
RF04 & 31.1 & 1.2 & 30.6 \\
RF05 & 0.0 & 0.0 & 0.0 \\
RF07 & 85.6 & 15.8 & 64.1 \\
RF08 & 44.1 & 1.9 & 23.7 \\
\hline
\end{tabular}



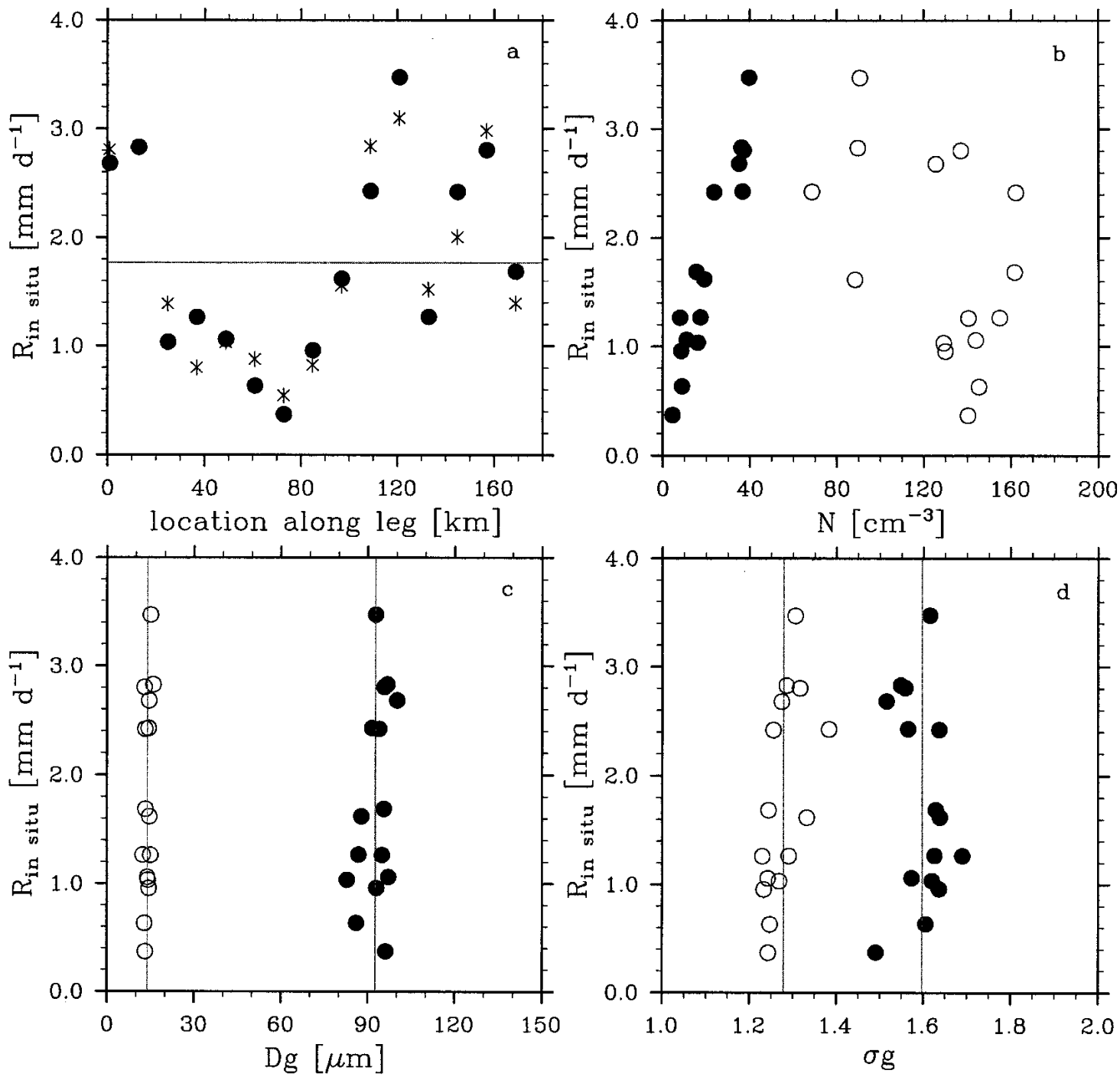

FIG. 12. Correlation between the in situ drizzle rate $R_{\text {in situ }}$ and the fitting parameters for the first cloud-base leg of RF07. (a) Drizzle rate $R_{\text {in situ }}$ in time (closed circles) and drizzle rate $R_{N}$ (asterisks) calculated as function of droplet concentration $N$ and the leg-averaged values of the geometric mean $D_{g}$ and the geometric standard deviation $\sigma_{g}$. The thin lines denote the leg-averaged value of both drizzle rates because they are nearly identical for this leg the lines lie on top of each other. (b) $R_{\text {in situ }}$ vs $N$ (note that the drizzle drop number is multiplied with a factor 1000 in order to fit both drop numbers in one plot), (c) $R_{\text {in situ }}$ vs $D_{g}$, and (d) $R_{\text {in situ }}$ vs $\sigma_{g}$. The data points based on the $260 \mathrm{X}$ are denoted by the closed circles; those based on the SPP-100 by the open circles. The leg-averaged values of $D_{g}$ and $\sigma_{g}$ used in the calculation of $R_{N}$ are shown by the thin lines in (c) and (d).

reaches the surface and plot this versus the depth of the subcloud layer in Fig. 14. Here our analysis is limited to time periods when we have simultaneous estimates of $R_{\text {radar,cb }}$ and $R_{\text {radar,sfc. }}$ Even for rather shallow subcloud layers most of the precipitation evaporates before reaching the surface, suggesting that the subcloud evaporation of drizzle is a strong function of depth below cloud base. Despite the spatial inhomogeneity of drizzle the leg-averaged evaporation values are fairly constant for each flight. The relatively less evaporation that occurs in some legs of RF02 (and to a lesser extent RF07 as well) may reflect the influence of humidified cores, that is, the correlation between subsegments of the leg with increased precipitation and increased humidity in the subcloud layer.

\section{Discussion}

It might seem perplexing that in section $4 \mathrm{c}$ we show that $R$ scales with $N$, yet in the $Z-R$ relationship scales as $Z^{2 / 3}$. For a lognormal distribution, one would expect that, if variations in $R$ are explained by variations in $N$, then $R$ should scale with $Z$ rather than with $Z^{2 / 3}$ (e.g., 


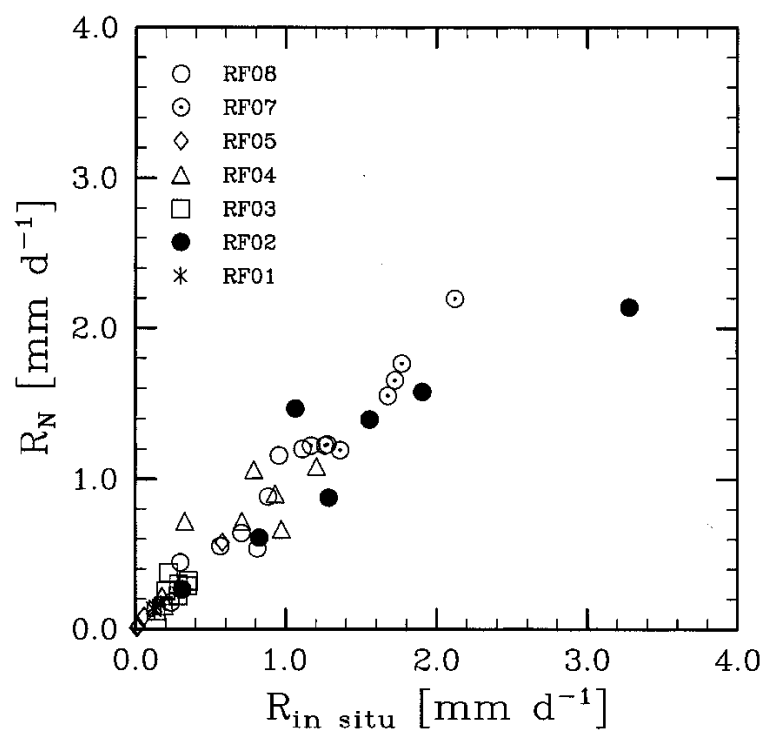

FIG. 13. Leg-averaged values of drizzle rate $R_{\text {in situ }}$ vs drizzle rate $R_{N}$ (see Fig. 12 for definition) for each flight.

Feingold and Levin 1986). For two reasons, this is not as contradictory as it seems. First, the other parameters in the lognormal distribution, that is, $D_{g}$ and $\sigma_{g}$, are not independent of $N$, as the simple argument which leads to the $R \propto Z$ scaling requires. Additionally, the tendency of $R$ to scale with $Z^{2 / 3}$ is based on log-space regressions, which weight points irrespective of their contribution to the net drizzle rate, that is, points that contribute negligibly count as much as points that weight more. The finding that $N$ variation explains

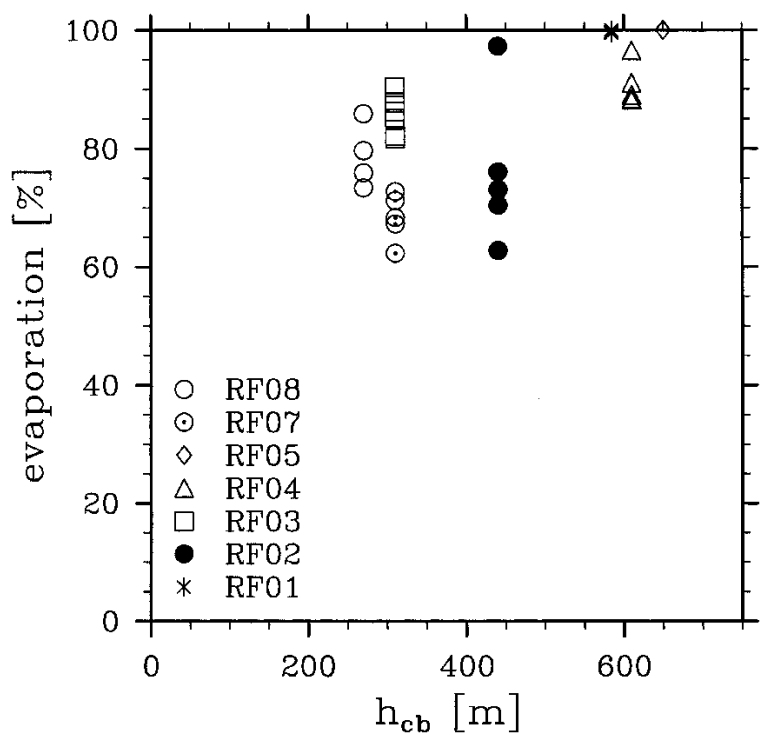

FIG. 14. Evaporation of drizzle in the subcloud layer defined as $\left(R_{\text {radar,cb }}-R_{\text {radar,sfc }}\right) / R_{\text {radar,cb }}$ as a function of mean cloud-base height $h_{\mathrm{cb}}$. Each point represents one flight leg of approximately $30 \mathrm{~min}$. most of the variability in $R$ is based on relations in linear space, which discount points whose contribution to the net $R$ are negligible.

These differences highlight just some of the difficulties in the $Z-R$ relationships that form the basis for the underlying precision of our analysis. Although we have tried to bound any uncertainty by a careful consideration of errors, it is also useful to investigate how sensitive our results are to some of the underlying assumptions, such as our decision to use extrapolated lognormals or our belief that it was best to tailor $Z-R$ relations on a flight by flight basis. To answer these questions we provide estimates of surface rain rates among flights using two alternative methods of analysis: no extrapolation of the lognormal functions and a generic $Z-R$ relationship for DYCOMS-II.

The "no-extrapolation" relationship was computed by determining the maximum diameter present in the measured DSD (with respect to every 2-min average) and using this value as an upper limit in our calculation of respective $R_{\text {in situ }}$ and $Z_{\text {in situ }}$. In Table 5 we present the flight-averaged surface drizzle rates computed with this $Z-R$ relationship. The $\langle R\rangle$ values are higher than in Table 1 (all within one standard deviation except RF07), with the largest increases going with the larger drizzle rates. The reason for this is that truncation of the spectra leads to higher values of both the slope $(n)$ and prefactor $(a)$ of the $Z-R$ relationship. With respect to $Z_{\text {in situ }}$ values up to roughly $0 \mathrm{~dB}_{Z}$ the relationships are within one standard deviation of the ones given in Table 2, while for higher $Z_{\text {in situ }}$ values they are within two standard deviations.

Table 5 also shows $R$ values derived using a $Z-R$ relationship based on all the nighttime flights (see Table 2). These values are lower than those in the first column of Table 5 but are within one standard deviation of the values in Table 1 . Inclusion of the daytime flight, RF08, in the derivation of the $Z-R$ relationship (see Table 2) would induce large changes, but based on just one flight it is not warranted to assign this to systematic day-night differences.

Both sensitivity tests-Table 1 versus Table 5-indi-

TABLE 5. Drizzle rates at 70-m height above the sea surface for the whole duration of each flight based on radar data. The conversion from radar reflectivity to drizzle rate is done with $Z-R$ relationships derived from in situ instruments (SPP-100 and 260X) without extrapolation of the lognormal fit for each individual flight and with one generic relationship (based on all nighttime flights).

\begin{tabular}{|c|c|c|}
\hline \multirow[b]{2}{*}{ Flight } & \multicolumn{2}{|c|}{ Drizzle rate } \\
\hline & $\begin{array}{l}\text { No extrapolation } \\
\left(\mathrm{mm} \mathrm{day}^{-1}\right)\end{array}$ & $\begin{array}{l}\text { Overall night } Z-R \\
\quad\left(\mathrm{~mm} \mathrm{day}^{-1}\right)\end{array}$ \\
\hline RF02 & $0.46 \pm 0.17$ & $0.27 \pm 0.04$ \\
\hline RF03 & $0.07 \pm 0.05$ & $0.06 \pm 0.01$ \\
\hline RF04 & $0.13 \pm 0.12$ & $0.11 \pm 0.01$ \\
\hline RF07 & $0.91 \pm 0.34$ & $0.73 \pm 0.09$ \\
\hline RF08 & $0.15 \pm 0.04$ & $0.09 \pm 0.01$ \\
\hline
\end{tabular}


cate that the specific assumptions for the conversion of radar reflectivity to drizzle rate do not unduly influence the basic conclusions in section 4 . The tests also indicate that the drizzle rates used in section 4 are likely to be conservative.

While it is clear that it is difficult to make judgements about the absolute accuracy of the drizzle rates, it is worth emphasizing that in situ and radar-derived values are in reasonable agreement (e.g., Figs. 3, 9, and 10). Another set of $Z-R$ relationships, derived using all incloud legs on a flight by flight basis, and using averages of observed droplet spectra (not fits) over 5-min periods yielded $R_{\text {radar,cb }}$ values about a factor of 2 above those of Table 3 and a factor of 5 higher for RF02. These differences are larger than the uncertainty estimates derived in section 3 in part due to not including in these latter estimates a stratification by height of the in-cloud spectra. Even so, the differences are (if RF02 is excepted) of the order of accuracy commonly connected with radar-derived rainfall rates. The fact that the drizzle rate presented here might be on the low side only strengthens our conclusion about the prevalence and importance of drizzle in stratocumulus.

\section{Summary}

We have analyzed microphysical data obtained during the Dynamics and Chemistry of Marine Stratocumulus II field study (DYCOMS-II). The field study consisted of nine flights with the NCAR/NSF C-130 aircraft in stratocumulus-topped boundary layers and took place in July 2001 in the northeastern Pacific westsouthwest of San Diego. Seven out of nine flights were flown during the night and seven out of nine flights consisted of a circular Lagrangian flight pattern. DYCOMSII was blessed with favorable conditions: relatively uniform, and spatially extensive stratocumulus cloud decks were probed with few breaks. Thus, the visually uniform-looking clouds hid rich differences in microphysical cloud structure.

The combined availability of the Wyoming cloud radar (Vali et al. 1998) and in situ microphysical instruments provided a unique opportunity to obtain estimates of drizzle rates in nocturnal marine stratocumulus. The results show that the prevalence of drizzle is higher than formerly thought. Out of seven flights analyzed, five had measurable mean precipitation at the surface, two with a substantial amount.

The drizzle rates have been estimated with respect to each flight, based on both in situ and remotely sensed data. Truncated lognormal functions have been fitted to the cloud droplet size distribution as measured by the SPP-100 and to the drizzle drop size distribution as measured by the 260X and 2DC. Based on those fits, the drizzle rate $R$ and reflectivity $Z$ values have been calculated analytically over a diameter range up to 1 $\mathrm{mm}$ for each leg. Next, $Z-R$ relationships were derived for each flight, both close to the surface and at cloud base height. Those relationships were used to convert $Z_{\text {radar }}$ data at those two height levels into a rain rate. The radar measured $Z$ close to the surface during all legs except the surface legs, so an almost continuous estimate of the surface drizzle rate could be obtained. In this way a more accurate flight-averaged drizzle rate can be estimated than one based on in situ data alone. Yet, in situ instruments are also able to characterize the amount of drizzle of a particular flight very well (i.e., indicate whether the drizzle rate is very light, moderate, or heavy), despite the inhomogeneity of the drizzle.

The general picture of drizzle in stratocumulus which emerges from the DYCOMS-II flights is one of large flight-averaged drizzle rates being mainly due to the occurrence of localized patches of strongly enhanced precipitation. Together with low flight-averaged drizzle rates correlating with a low intensity of drizzle this strongly suggests that drizzle could induce a transition in cloud structure (Stevens et al. 1998; Paluch and Lenschow 1991). Variability in drizzle rates among flights correlates well with cloud depth cubed divided by the total cloud droplet number, while variability in the in situ drizzle rate within each flight is explained by the variability in total drizzle drop number. Thus higher precipitation rates are not due to a change in shape of the drizzle drop distribution but are mainly caused by the more frequent occurrence of larger drizzle dropsin the right tail end of the distribution-as a consequence of the higher total number of drizzle drops. Consequently, leg-averaged in situ drizzle rates are well represented by values calculated by using leg-averaged droplet distributions. On the macroscopic scale the drizzle rate also correlates negatively with $\Delta T$ (the difference between the $11-\mu \mathrm{m}$ and $14-\mu \mathrm{m}$ brightness temperatures as measured by the GOES-10 satellite) and shows signs as well of thresholdlike dependence on in situ cloud-top effective radius. Evaporation of drizzle in the subcloud layer is rather high, even for shallow boundary layers, and displays only a weak dependence on the depth of this layer.

Acknowledgments. Support by the National Science Foundation, through Grants ATM 0097053 and ATM$0094956(\mathrm{GV})$ is gratefully acknowledged. For the first author this work is part of the research programe of the Stichting voor Fundamenteel Onderzoek der Materie (FOM), which is financially supported by the Nederlandse Organisatie voor Wetenschappelijk Onderzoek (NWO).

\section{APPENDIX}

\section{Fitting Procedures}

The first step in the fitting procedure is to calculate $D_{g}$ and $\sigma_{g}$ and $N$ (for definition of these terms see section $3 a$ ) from the data directly. The lognormal func- 
tion specified by these values, however, is biased towards the higher moments and, in general, a better fit can be obtained by taking into account that the measured DSDs are truncated at both ends (defined as $D_{\text {min }}$ and $\left.D_{\max }\right)$. The so-called truncated lognormal fit can be defined as the lognormal function having the same $D_{g}$ and $\sigma_{g}$ and $N$ between $D_{\min }$ and $D_{\text {max }}$ as the data. In Feingold and Levin (1986) analytical relationships are given between $D_{g}$ and $\sigma_{g}$ of the nontruncated lognormal function and the truncated lognormal function. To show the goodness of a certain fit it is common to use the so-called $\chi^{2}$ test: $\chi^{2}$ is defined as the ratio between the variance of the fit $s^{2}$ and the variance of the data $\sigma^{2}$ multiplied by the degrees of freedom $\nu=n-m$, with $m$ the number of parameters (in our case equal to 3 ) used to fit a function to $n$ data points.

The variance of the fit $s^{2}$ is given by

$$
s^{2}=\frac{1}{\nu} \sum \frac{1 / \sigma_{i}^{2}}{1 / N \Sigma 1 / \sigma_{i}^{2}}\left[N_{i}-f\left(D_{i}\right)\right]^{2},
$$

with $i$ the index representing the bin number of the DSD. Once the $\chi^{2}$ value is known, a statistical measure of goodness of fit can be determined by assuming that the errors in the data points are normally distributed. A rough estimate whether a fit is statistically "good" or not can be obtained by comparing $\chi^{2}$ and $\nu$; for comparable values of the two the fit is acceptable. The applied length of the intermediate averaging period of 20 $\mathrm{s}$ is based upon this because $\sigma^{2}$ calculated from six 20-s averages ensures a statistically good fit. Taking the standard deviation based upon one averaging period of $120 \mathrm{~s}$ gives a value for $\chi^{2}<<1$, indicating an overestimation of the variance in the data (Bevington and Robinson 1992).

Besides a statistical measure of the goodness of fit, uncertainty estimates for the fitting parameters should be given. We determined rough estimates of those uncertainties by variation of $\chi^{2}$ round its local minimum:

$$
\sigma=\Delta a \sqrt{\frac{2}{\chi_{1}^{2}-2 \chi_{2}^{2}+\chi_{3}^{2}},}
$$

with $a$ one of the fitting parameters and $\chi_{i}^{2}$ the value of $\chi^{2}$ for $a_{1}, a_{2}=a_{1}+\Delta a$ and $a_{3}=a_{2}+\Delta a$. It is important to realize that this uncertainty estimate $\sigma$ should be interpreted as the variation needed in $a$ to increase the minimum value of $\chi^{2}$ by 1 . (Bevington and Robinson 1992).

So after the calculation of the truncated fitting parameters, the value of $\chi^{2}$ is calculated, together with an estimate of the uncertainty in the three fitting parameters. The calculations of the uncertainties in the parameters supply six other $\chi^{2}$ values; comparison of these values show that the truncated lognormal fit is often (more often for the CDSDs than for the DDSDs) but not always the fit with the lowest value for $\chi^{2}$, that is, the best fit available. Despite this, the values of the fitting parameters of the truncated lognormal fit are taken because it is the fit that conserves the moments of the data, and in general the values of the fitting parameters would vary only slightly if the absolute best fit was taken. (Another reason not to always trust the $\chi^{2}$ method is the fact that in the derivation of the least squares method the assumption is made that uncertainties in the data are normally distributed, which may not be the case for the DSD which might suffer from more under sampling at the larger bin sizes.)

Because drizzle drops have a small incident rate some caution is necessary when fitting a DSD. In order to ensure enough counts in a sufficient number of bins the 1-s data of the SPP-100, 260X, and the 2DC are averaged over $120 \mathrm{~s}$. The length of this period is an optimum between reduction of the sampling error and conservation of the temporal and spatial scales. The lower limit for the necessary number of counts in one bin is five, this number is often taken as one of the requirements to be able to classify a distribution as Gaussian instead of Poisson. However, bins with 4 counts or less are not disregarded despite the violation of the Gaussian distribution assumption. This is justified because almost all the bins with 4 counts or less are for the larger drop diameters and the fitting parameters are not sensitive to leaving out quite a number of bins of the largest drops. On the other hand the parameters are sensitive to the opposite; reducing the number of bins by removing bins with small drop diameters. This is easily understood when one realizes that the bins with small diameter generally contain the most counts, thus contributing most to the moments of the DSD. In order to fit two lognormal functions to the two physical modes instead of to the DSDs of the two different instruments, the fit to the CDSD is subtracted from the data of the DDSD (only for the region in which the measurements overlap) before fitting the latter.

During the fitting procedure bad convergence of the fit is encountered on several occasions. To deal with this several steps are taken. First of all, the data is always checked for the presence of enough bins for a fit; all DSDs with less than 10 bins containing data are disregarded. If there are enough bins but the convergence is bad, adjustments are made to the left or right limit because sometimes the behavior at the DSD limits complicates the fitting. In all cases the fitting procedure is disregarded if the number of bins becomes too small to ensure a good fit. And if the convergence is still unsatisfactorily after these two corrections, the fitting procedure is abandoned and no fit is calculated.

\section{REFERENCES}

Ackerman, A. S., O. B. Toon, and P. V. Hobbs, 1993: Dissipation of marine stratiform clouds and collapse of the marine boundary layer due to the depletion of cloud condensation nuclei by clouds. Science, 262, 226-229.

Albrecht, B. A., 1989: Aerosols, cloud microphysics, and fractional cloudiness. Science, 245, 1227-1230. 
Aldous, D., 1999: Deterministic and stochastic models for coalescence (aggregation, coagulation): A review of the mean-field theory for probabilists. Bernoulli, 5, 3-48.

Austin, P., Y. Wang, R. Pincus, and V. Kujala, 1995: Precipitation in stratocumulus clouds: Observational and modeling results. J. Atmos. Sci., 52, 2329-2352.

Bevington, P. R., and D. K. Robinson, 1992: Data Reduction and Data Analysis for the Physical Sciences. McGraw-Hill, 328 pp.

Boers, R., J. B. Jensen, and P. B. Krummel, 1996: Microphysical and short-wave radiative structure of wintertime stratocumulus over the Southern Ocean. Quart. J. Roy. Meteor. Soc., 122, $1307-1339$.

— - _ and _ 1998: Microphysical and short-wave radiative structure of stratocumulus over the Southern Ocean: Summer results and seasonal differences. Quart. J. Roy. Meteor. Soc., 124, 151-168.

Brenguier, J. L., T. Bourrianne, A. de Araujo Coelho, R. J. Isbert, R. Peytavi, D. Trevarin, and P. Weschler, 1998: Improvements of droplet distribution size measurements with the fast-FSSP (forward scattering spectrometer probe). J. Atmos. Oceanic Technol., 15, 1077-1090.

Bretherton, C. S., P. Austin, and S. T. Siems, 1995: Cloudiness and marine boundary layer dynamics in the ASTEX Lagrangian experiment. Part II: Cloudiness, drizzle, surface fluxes, and entrainment. J. Atmos. Sci., 52, 2724-2735.

Brost, R. A., J. C. Wyngaard, and D. H. Lenschow, 1982: Marine stratocumulus layers. Part II: Turbulence budgets. J. Atmos. Sci., 39, 818-836.

Chen, C., and W. R. Cotton, 1987: The physics of the marine stratocumulus mixed layer. J. Atmos. Sci., 44, 2951-2977.

Duynkerke, P. G., H. Zhang, and P. J. Jonker, 1995: Microphysical and turbulent structure of nocturnal stratocumulus as observed during ASTEX. J. Atmos. Sci., 52, 2763-2777.

_ , and Coauthors, 1999: Intercomparison of three- and onedimensional model simulations and aircraft observations of stratocumulus. Bound.-Layer Meteor., 92, 453-487.

Feingold, G., and Z. Levin, 1986: The lognormal fit to raindrop spectra from frontal convective clouds in Israel. J. Climate Appl. Meteor., 25, 1346-1363.

- B. Stevens, W. R. Cotton, and A. S. Frisch, 1996: The relationship between drop in-cloud residence time and drizzle production in numerically simulated stratocumulus clouds. $J$. Atmos. Sci., 53, 1108-1122.

Ferek, R. J., and Coauthors, 2000: Drizzle suppression in ship tracks. J. Atmos. Sci., 57, 2707-2728.

Frisch, A. S., C. W. Fairall, and J. B. Snider, 1995: Measurement of stratus cloud and drizzle parameters in ASTEX with a K-band doppler radar and a microwave radiometer. J. Atmos. Sci., 52, 2788-2799.

Gerber, H., 1994: New microphysics sensor for aircraft use. Atmos. Res., 31, 235-252.

_ 1996: Microphysics of marine stratocumulus clouds with two drizzle modes. J. Atmos. Sci., 53, 1649-1662.

Haddad, Z. S., S. L. Durden, and E. Im, 1996: Parameterizing the raindrop size distribution. J. Appl. Meteor., 35, 2680-2688.

Han, Q., W. Rossow, R. Welch, A. White, and J. Chou, 1995: Validation of satellite retrievals of cloud microphysics and liquid water path using observations from FIRE. J. Atmos. Sci., 52, 4183-4195.
Hocking, L. M., 1959: The collision efficiency of small drops. Quart. J. Roy. Meteor. Soc., 85, 44-53.

Khairoutdinov, M., and Y. Kogan, 2000: A new cloud physics parameterization in a large-eddy simulation model of marine stratocumulus. Mon. Wea. Rev., 128, 229-243.

Kogan, Y. L., M. P. Khairoutdinov, D. K. Lilly, Z. N. Kogan, and Q. Liu, 1995: Modeling of stratocumulus cloud layers in a large eddy simulation model with explicit microphysics. $J$. Atmos. Sci., 52, 2923-2940.

Kraus, E. B., 1963: The diurnal precipitation change over the sea. J. Atmos. Sci., 20, 551-556.

Lasher-Trapp, S. G., W. A. Cooper, and A. M. Blyth, 2002: Measurement of ultragiant aerosol particles in the atmosphere from the small cumulus microphysics study. J. Atmos. Oceanic Technol., 19, 402-408.

Nicholls, S., 1984: The dynamics of stratocumulus: Aircraft observations and comparisons with a mixed layer model. Quart. J. Roy. Meteor. Soc., 110, 783-820.

_ 1987: A model of drizzle growth in warm, stratiform clouds. Quart. J. Roy. Meteor. Soc., 113, 1141-1170.

- and J. Leighton, 1986: An observational study of the structure of stratiform cloud sheets. Part I: Structure. Quart. J. Roy. Meteor. Soc., 112, 431-460.

Paluch, I. R., and D. H. Lenschow, 1991: Stratiform cloud formation in the marine boundary layer. J. Atmos. Sci., 48, 21412158.

Pawlowska, H., and J.-L. Brenguier, 2003: An observational study of drizzle formation in stratocumulus clouds for general circulation model (GCM) parameterizations. J. Geophys. Res., 108, 8630, doi:10.1029/2002JD002679.

Perez, J. C., F. Herrera, F. Rosa, A. Gonzales, M. Wetzel, R. Borys, and D. Lowenthal, 2000: Retrieval of marine stratus cloud droplet size from NOAA AVHRR night imagery. $R e$ mote Sens. Environ., 73, 31-45.

Pincus, R., and M. B. Baker, 1994: Effect of precipitation on the albedo susceptibility of clouds in the marine boundary layer. Nature, 372, 250-252.

Rogers, R. R., and M. K. Yau, 1989: A Short Course in Cloud Physics. Butterworth Heineman, 290 pp.

Stevens, B., W. R. Cotton, G. Feingold, and C. H. Moeng, 1998: Large-eddy simulations of strongly precipitating, shallow, stratocumulus-topped boundary layers. J. Atmos. Sci., 55, 3616-3638.

—, and Coauthors, 2003: Dynamics and chemistry of marine stratocumulus-DYCOMS-II. Bull. Amer. Meteor. Soc., 84, 579-593.

, G. Vali, K. Comstock, R. Wood, M. C. Zanten, P. Austin, C. S. Bretherton, and D. H. Lenschow, 2005: Pockets of open cells (POCs) and drizzle in marine stratocumulus. Bull. Amer. Meteor. Soc., in press.

Vali, G., R. D. Kelly, J. French, S. Haimov, D. Leon, R. E. McIntosh, and A. Pazmany, 1998: Finescale structure and microphysics of coastal stratus. J. Atmos. Sci., 55, 3540-3564.

Wang, S., and Q. Wang, 1994: Roles of drizzle in a onedimensional third-order turbulence closure model of nocturnal stratus-topped marine boundary layer. J. Atmos. Sci., 51, 1559-1576. 